\title{
BPS Quivers of Five-Dimensional SCFTs, Topological Strings and q-Painlevé Equations
}

\author{
Giulio Bonelli, Fabrizio Del Monte® and Alessandro Tanzini
}

\begin{abstract}
We study the discrete flows generated by the symmetry group of the BPS quivers for Calabi-Yau geometries describing five-dimensional superconformal quantum field theories on a circle. These flows naturally describe the BPS particle spectrum of such theories and at the same time generate bilinear equations of q-difference type which, in the rank one case, are q-Painlevé equations. The solutions of these equations are shown to be given by grand canonical topological string partition functions which we identify with $\tau$-functions of the cluster algebra associated to the quiver. We exemplify our construction in the case corresponding to five-dimensional $S U(2)$ pure super Yang-Mills and $N_{f}=2$ on a circle.
\end{abstract}

\section{Contents}

1. Introduction

2. BPS Spectrum of 5d SCFT on $S^{1}$ and Quiver Mutations 2726

2.1. Super Yang-Mills, $k=0$

2.2. Super Yang-Mills, $k=1$

2.3. $N_{f}=2, k=0$

3. Discrete BPS Quiver Dynamics, Cluster Algebras and q-Painlevé Equations

3.1. Cluster Algebras and Quiver Mutations

3.2. Pure Gauge Theory and q-Painlevé $\mathrm{III}_{3}$

3.3. Super Yang-Mills with Two Flavors and qPIII

3.4. Super Yang-Mills with Two Flavors, q-Painlevé IV and qPainlevé II

3.5. Summary of $\mathrm{dP}_{3}$ Bilinear Equations 
4.1. Local $\mathbb{F}_{0}$ and $\mathrm{qPIII}_{3}$

4.2. q-Painlevé $\mathrm{III}_{1}$ and Nekrasov Functions

5. Degeneration of Cluster Algebras and Four-Dimensional Gauge Theory

5.1. From local $\mathbb{F}_{0}$ to the Kronecker Quiver

5.2. Local $\mathbb{F}_{1}$

5.3. Local $\mathrm{dP}_{3}$

A. q-Special Functions and Nekrasov Functions

B. q-Painlevé III and IV in Tsuda's Parameterization

B.2. qPIV

\section{Introduction}

A crucial problem in quantum field theory (QFT) is understanding its nonperturbative aspects in the concrete terms of exact computations. QFTs can be embedded in string theory/M-theory via geometric engineering [1]. Specifically, it can be obtained as the low energy limit of a compactified string theory in a large volume limit, which is needed to decouple its gravitational sector. When QFT is obtained in this way, the nature of exact dualities gets unveiled through the geometric properties of the string theory background behind it: the string theory on the non-compact Calabi-Yau (CY) background geometry encodes the spectral geometry of integrable systems whose solutions allow to obtain exact results. This is possible because the non-perturbative sector of string theory, described by D-branes, gets transferred through this procedure to the geometrically engineered QFT. The set-up engineered by M-theory compactification on $C Y_{3} \times S^{1}$, in the limit of large $C Y_{3}$ volume and finite $S^{1}$ radius, is that of a five-dimensional supersymmetric QFT on a circle, whose particles arise from membranes wrapped on the 2-cycles of a suitable non-compact CY manifold. As such, the counting of the BPS protected sectors of the theory can be obtained by considering a dual picture given in terms of a topological string on $\mathrm{CY}_{3}$. The precise dictionary between the two descriptions is obtained by identifying the topological string partition function on the $C Y_{3}$ with the supersymmetric index of the gauge theory, which is conjectured to capture the exact BPS content of its 5d SCFT completion [2]. More generally, the supersymmetric index of the gauge theory with surface defects is matched with the corresponding D-brane open topological string wave function [3,4]. The coupling constants and the moduli of the QFT arise from the geometric engineering as CY moduli parameters (Kähler and complex in the A and B-model picture, respectively). Therefore, the QFT generated in this way is naturally in a generic phase in which all the coupling constants can be finite. To identify 
the weakly coupled regimes, one has to consider particular corners in the CY moduli space. In such corners, the topological string theory amplitudes allow a power expansion in at least one small parameter which is identified with the gauge coupling, while the others are fugacities of global symmetries of the QFT (masses and Coulomb parameters).

The problem we would like to face, in the general set-up described so far, is that of understanding how to predict the properties of such a supersymmetric index, given the non-compact CY manifold which realizes the five-dimensional theory via geometric engineering. We will show that this index satisfies suitable q-difference equations which in the rank one case, namely for Calabi-Yau whose mirror is a local genus one curve, are well known in the mathematical physics literature as q-Painlevé equations [5]. These are classified in terms of their symmetry groups as in Fig. 1. Remarkably, this classification coincides with the one obtained from string theory considerations in [6]. This allows to describe the grand canonical partition function of topological strings as $\tau$-functions of a discrete dynamical system, whose solutions encode the BPS spectrum of the theory. From this viewpoint, the grand canonical partition function is actually vector-valued in the symmetry lattice of the discrete dynamical system at hand. The exact spectrum of the relevant integrable system can be computed from the zeroes of the grand partition function.

The solutions of the discrete dynamical system are naturally parameterized in different ways according to the different BPS chambers of the theory. We will show that the Nekrasov-Okounkov [7] presentation of the supersymmetric index can be recovered in the large volume regions of the Calabi-Yau moduli space which allow the geometric engineering of five-dimensional gauge theories. The expansion parameter is schematically $e^{-V}, V$ being the volume of the relevant cycle corresponding to the instanton counting parameter. Around the conifold point, the solution is instead naturally parameterized in terms of a matrix model providing the non-perturbative completion of topological string via topological string/spectral theory correspondence [8]. The case of local $\mathbb{F}_{0}$ geometry, which engineers pure $S U(2)$ Yang-Mills in five dimensions at zero Chern-Simons level, was discussed in detail in [9]. In this case, the matrix model is a q-deformation of the $O(2)$ matrix model describing $2 \mathrm{~d}$ Ising correlators $[10,11]$. The quantum integrable system arising from the quantum Calabi-Yau geometry is two-particle relativistic Toda chain.

In this paper, we show that the discrete dynamics is determined from the analysis of the extended automorphism group of the BPS quiver associated to the Calabi-Yau geometry. In this respect let us recall the results [12-15] where the BPS state spectrum of a class of four-dimensional supersymmetric theories is generated through quiver mutations. The quiver describes the BPS vacua of the supersymmetric theory and encodes the Dirac pairing among the stable BPS particles. The consistency of the Kontsevich-Soibelman formula [16] for the wall-crossing among the different stability chambers is encoded in Y- and Q- systems of Zamolodchikov type. While this program has been mostly studied for four-dimensional theories, recently a proposal for BPS quivers for the five-dimensional theory on a circle has been advanced in [17]. The 
five-dimensional BPS quivers are conjectured to describe the BPS spectrum of the five-dimensional theory on $\mathbb{R}^{4} \times S^{1}$ and have two extra nodes with respect to the corresponding four-dimensional ones, representing, in properly chosen regimes, the KK tower of states and the five-dimensional instanton monopole which characterize the theory on a circle.

The proposal we make in this paper is that these very same quivers also encode the q-difference equations satisfied by the SUSY index. These are generated by studying the application of extended quiver symmetries on the relevant cluster algebra variables $\tau$, the latter being identified with a vectorvalued topological string grand partition function. The action of the symmetry generators on the cluster algebra coefficients $y$ keeps track of the discrete flows for the $\tau$-functions. As such, once the gauge theory is considered on a self-dual $\Omega$-background, we obtain that its supersymmetric index satisfies a proper set of q-Painlevé equations generated by the extended automorphisms of the quiver. More precisely, we identify different dynamics corresponding to different generators of the extended automorphism group. In a given patch, in which the topological string theory engineers a weakly coupled five-dimensional theory, the generator shifting the chosen gauge coupling induces the q-Painlevé dynamics, while the other independent ones act as Bäcklund transformations of the former.

In this paper, we make a first step towards realizing the above proposal by showing that the discrete flows induced by the extended automorphism group on the BPS quiver generate in a simple way the full BPS spectrum of the $5 \mathrm{~d}$ SCFT for some examples in the rank one case. At the same time we show that the Nekrasov-Okounkov dual partition function of the $5 \mathrm{~d}$ gauge theory obtained by relevant deformation of those theories solves the q-Painlevé equations associated to the same discrete flows. This will be accompanied also by the study of the degeneration of the five-dimensional cluster algebra into the four-dimensional one by appropriate decoupling limits. More specifically, we explore the above connection by considering in detail the case of pure $S U(2)$ gauge theory, engineered by local $\mathbb{F}_{0}$ and local $\mathbb{F}_{1}$ depending on the value of the Chern-Simons level, as well as the $S U(2)$ gauge theory with two fundamental flavors, or equivalently the one engineered by the local Calabi-Yau threefold over $d P_{3}$. This case gives a much richer lattice of bilinear equations than the case of pure gauge, with four independent discrete time evolutions.

It was noticed in [18-20] that cluster algebras provide a natural framework to describe q-deformed Painlevé equations, together with their higher rank generalizations and quantization (crucial to describe the refined topological string set up). Further, following the results of $[9,21]$ evidence was provided for the identification of the q-Painlevé tau function with Topological String partition function on toric Calabi-Yau threefold, or q-deformed conformal blocks. However, while the connection with q-Painlevé equations was derived in many cases, only in the case of pure $S U(N)$ gauge theory (corresponding in the $S U(2)$ case to q-Painlevé $\mathrm{III}_{3}$ ) bilinear equations were derived from the cluster algebra. In this paper, we derive from the cluster algebra bilinear equations for the $S U(2)$ theory with two flavors, as well as a bilinear form 


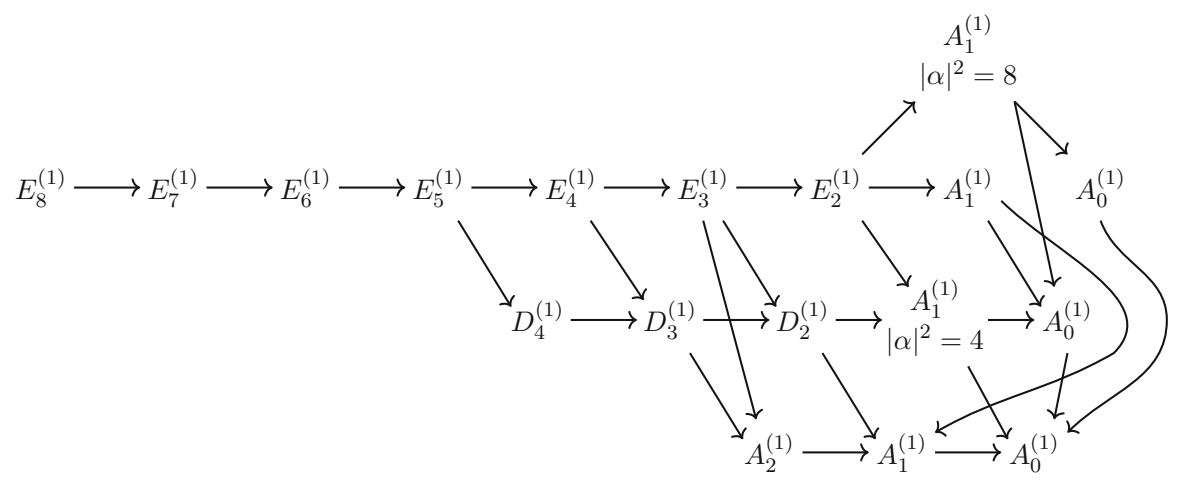

Figure 1. Sakai's classification of discrete Painlevé equations by symmetry type

the q-Painlevé IV equation from the cluster algebra of the local $d P_{3}$ geometry which to our knowledge did not appear in the literature, and we discuss its physical interpretation in terms of the $\left(A_{1}, D_{4}\right)$ Argyres-Douglas theory.

Notice that, given the geometrical datum of the toric Calabi-Yau, it is possible to obtain its associated quiver from the corresponding dimer model $[22,23]$, and the A-cluster variables defined from this quiver lead to bilinear equations. In many cases these have been shown to be satisfied by dual partition functions of Topological String theory on this same Calabi-Yau [24], or by q-deformed Virasoro conformal blocks [21,25-27]. These can also be rephrased in terms of K-theoretic blowup equations [24,28,29].

Here is the plan of the paper. In Sect. 2 we discuss BPS quiver spectroscopy, by first checking our perspective with the known case of local $\mathbb{F}_{0}$ which correctly reproduces the results of [17], before turning to discuss the new cases of local $\mathbb{F}_{1}$ and $d P_{3}$ five-dimensional gauge theories. In Sect. 3 we study the discrete BPS quiver dynamics for the above examples and the related cluster algebras and obtain the explicit q-Painlevé equations in bilinear form. In Sect. 4 we show that indeed the $\tau$-functions of the specific q-Painlevé flows can be matched with shifted Nekrasov-Okounkov partition function of the corresponding gauge theory. In Sect. 5 we make more precise the observation that the four-dimensional quiver can be obtained by removing two nodes from the five-dimensional one, and show, for all the examples previously considered that appropriate scaling limits of the cluster variables correctly reproduce the cluster algebra of the four-dimensional subquiver, which is known to describe the 4d BPS spectrum. In "Appendix A" we collect all the necessary formulas for five-dimensional Nekrasov functions, while in "Appendix B" we show how the bilinear equations of [30] can be also recovered from the cluster algebra approach. These are seemingly different from the ones of [26], but we show that they correspond to a different choice of initial coefficients for the cluster algebra. "Appendix C" collects few technical points related to improved saddle point expansion of NO partition functions used in the paper. 


\section{BPS Spectrum of 5d SCFT on $S^{1}$ and Quiver Mutations}

The construction that generates the BPS spectrum of a supersymmetric theory through mutations of its BPS quiver is known as mutation algorithm, and was widely employed in the case of four-dimensional $\mathcal{N}=2$ theories $[13,14,31]$. We recall that, given a quiver with adjacency matrix $B_{i j}$, the mutation at its $k$ th node $^{1}$ is defined by

$$
\mu_{k}\left(B_{i j}\right)= \begin{cases}-B_{i j}, & i=k \text { or } j=k, \\ B_{i j}+\frac{B_{i k}\left|B_{k j}\right|+B_{k j}\left|B_{i k}\right|}{2}, & \end{cases}
$$

The mutations of the BPS charges $\gamma_{i}$ are given by

$$
\mu_{k}\left(\gamma_{j}\right)= \begin{cases}-\gamma_{j}, & j=k, \\ \gamma_{j}+\left[B_{k j}\right]_{+} \gamma_{k}, & \text { otherwise. }\end{cases}
$$

where we defined $[x]_{+}=\max (x, 0)$. In this context, each node of the quiver represents a BPS charge in the upper-half plane, and a mutation $\mu_{k}$ encodes the rotation of a BPS ray vector out of the upper half central charge Z-plane (see $[13,31]$ for a detailed description) in counterclockwise sense. If the charge is rotated out of the upper-half plane clockwise instead, one has to use a slightly different mutation rule

$$
\widetilde{\mu_{k}}\left(\gamma_{j}\right)= \begin{cases}-\gamma_{j}, & j=k, \\ \gamma_{k}+\left[-B_{k j}\right]+\gamma_{k}, & \text { otherwise. }\end{cases}
$$

This construction is most effective when the BPS states lie in a "finite chamber", i.e. when the BPS spectrum consists entirely of hypermultiplets. This is not the case for the $5 \mathrm{~d}$ theories we are considering: due to the intrinsically stringy origin of the UV completion of these theories, in general the BPS spectrum is organized in Regge trajectories of particles with arbitrary higher spin $[32,33]$; such chambers of the moduli space are known as "wild chambers". In [17] an argument was put forward for the existence of a "tame chamber" of the moduli space. Such a region is characterized by the fact that the higherspin particles are unstable and decay, and one is left with hypermultiplet and vector multiplets only, giving a situation much similar to the four-dimensional weakly coupled chambers.

\subsection{Super Yang-Mills, $k=0$}

As an example, Closset and Del Zotto argued that the spectrum for local $\mathbb{F}_{0}$, engineering pure $S U(2) S Y M$ on $\mathbb{R}^{4} \times S^{1}$ with Chern-Simons level $k=0$, in such a tame chamber is organized as two copies of the weakly coupled chamber of the four-dimensional pure $S U(2)$ gauge theory. The relevant quiver is depicted in Fig. 2, and its adjacency matrix is

\footnotetext{
${ }^{1}$ This is an example of a cluster algebra structure that we will introduce more thoroughly in Sect. 3.
} 


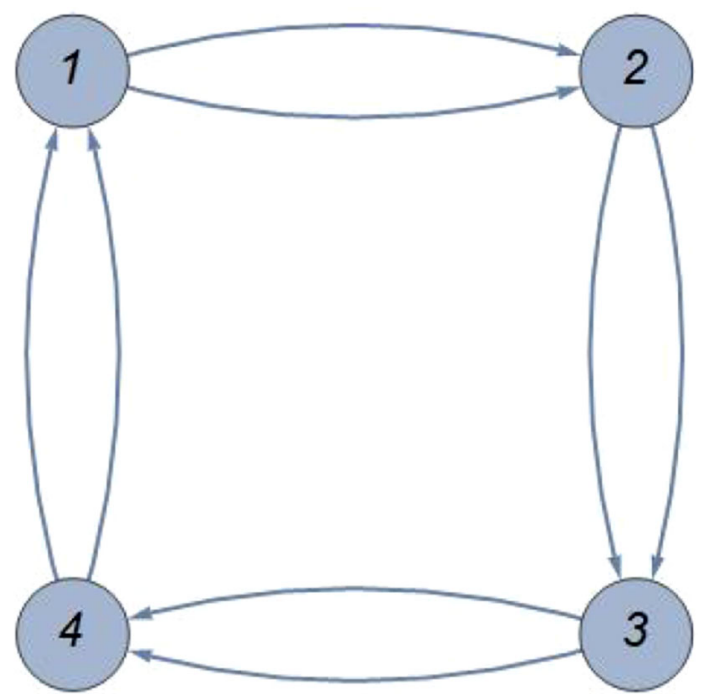

Figure 2. Quiver associated to local $\mathbb{F}_{0}$

$$
B=\left(\begin{array}{cccc}
0 & 2 & 0 & -2 \\
-2 & 0 & 2 & 0 \\
0 & -2 & 0 & 2 \\
2 & 0 & -2 & 0
\end{array}\right)
$$

The spectrum of this theory was originally derived by using the mutation algorithm in [17], by applying the sequence of mutations

$$
\mathbf{m}=\mu_{2} \mu_{4} \mu_{3} \mu_{1}
$$

to the BPS charges. Each mutation in this context describes the change of elementary BPS states under a change of duality frame, so that the transformation $\mathbf{m}$ amounts to the action of a self-duality on the BPS spectrum. The n-th iteration of this operator has the following effect on the charges $\gamma_{i}$, $i=1, \ldots, 4$ of the BPS states:

$$
\begin{array}{ll}
\mathbf{m}^{n}\left(\gamma_{1}\right)=\gamma_{1}+2 n \delta_{u}, & \mathbf{m}^{n}\left(\gamma_{2}\right)=\gamma_{2}-2 n \delta_{u}, \\
\mathbf{m}^{n}\left(\gamma_{3}\right)=\gamma_{3}+2 n \delta_{d}, & \mathbf{m}^{n}\left(\gamma_{4}\right)=\gamma_{4}-2 n \delta_{d},
\end{array}
$$

with

$$
\delta_{u}=\gamma_{1}+\gamma_{2}, \quad \delta_{d}=\gamma_{3}+\gamma_{4}
$$

The action of $\mathbf{m}$ corresponds to rotating out of the upper-half plane the BPS charges in the order 1342 . The towers of states obtained in this way accumulate on the vector multiplets from one side only. Because of this, the operator $\mathbf{m}$ is not sufficient: in order to construct the full spectrum in this chamber, it is necessary to use also the second operator

$$
\hat{\mathbf{m}}=\hat{\mu}_{1} \hat{\mu}_{3} \hat{\mu}_{2} \hat{\mu}_{4},
$$




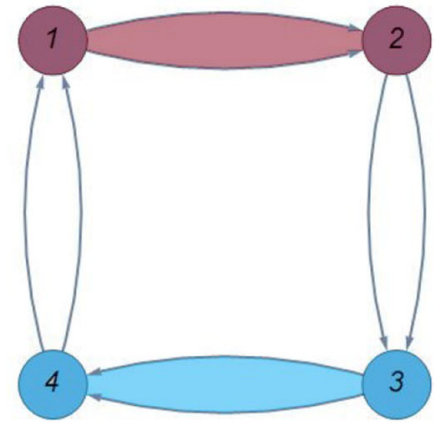

(a) Subquiver decomposition for $T_{\mathbb{F}_{0}}$

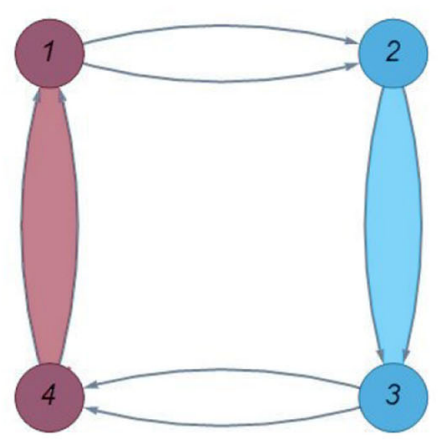

(b) Subquiver decomposition for $T_{\mathbb{F}_{0}}^{\prime}$

Figure 3. a Subquiver decomposition for $T_{\mathbb{F}_{0}}$. b Subquiver decomposition for $T_{\mathbb{F}_{0}}^{\prime}$

constructed from right mutations (2.3). The shifts obtained from this operator are

$$
\begin{array}{ll}
\hat{\mathbf{m}}^{n}\left(\gamma_{1}\right)=\gamma_{1}-2 n \delta_{u}, & \hat{\mathbf{m}}^{n}\left(\gamma_{2}\right)=\gamma_{2}+2 n \delta_{u}, \\
\hat{\mathbf{m}}^{n}\left(\gamma_{3}\right)=\gamma_{3}-2 n \delta_{d}, & \hat{\mathbf{m}}^{n}\left(\gamma_{4}\right)=\gamma_{4}+2 n \delta_{d} .
\end{array}
$$

The resulting BPS spectrum consists of two vector multiplets $\delta_{u}, \delta_{d}$, and two towers of hypermultiplets

$$
\gamma_{1}+n \delta_{u}, \quad \gamma_{2}+n \delta_{u}, \quad \gamma_{3}+n \delta_{d}, \quad \gamma_{4}+n \delta_{d}
$$

These are two copies of the weakly coupled spectrum of four-dimensional $\mathcal{N}=2 S U(2)$ pure SYM, which can be thought as being associated to the decomposition of the quiver in Fig. 2 into two four-dimensional Krönecker subquivers, as depicted in Fig. 3a.

Let us show an alternative derivation of the above result, making use of the group $\mathcal{G}_{Q}$ of quiver automorphisms. This contains the semidirect product $D i h_{4} \ltimes W\left(A_{1}^{(1)}\right)$, where $D i h_{4}$ is the dihedral group of the square, which consists only of permutations. The automorphisms group is generated by

$$
\pi_{1}=(1,3) \iota, \quad \pi_{2}=(4,3,2,1), \quad T_{\mathbb{F}_{0}}=(1,2)(3,4) \mu_{1} \mu_{3} .
$$

The operator $T_{\mathbb{F}_{0}}$ is a Weyl translation on the $A_{1}^{(1)}$ lattice.

This operator directly generates the whole BPS spectrum of the theory by acting on the charges. Indeed, by applying the mutation rules (2.2) we obtain

$$
\begin{aligned}
T_{\mathbb{F}_{0}}^{n}\left(\gamma_{1}\right)=\gamma_{1}+n \delta_{u}, & T_{\mathbb{F}_{0}}^{n}\left(\gamma_{2}\right)=\gamma_{2}-n \delta_{u}, \\
T_{\mathbb{F}_{0}}^{n}\left(\gamma_{3}\right)=\gamma_{3}+n \delta_{d} & T_{\mathbb{F}_{0}}^{n}\left(\gamma_{4}\right)=\gamma_{4}-n \delta_{d} .
\end{aligned}
$$

By computing the Dirac pairing of these states, which is given by the adjacency matrix of the quiver, in particular

$$
\left\langle\delta_{u}, \delta_{d}\right\rangle=\delta_{u}^{T} \cdot B \cdot \delta_{d}=0
$$


we see that $T_{\mathbb{F}_{0}}$ generates mutually local towers of states

$$
\gamma_{1}+n \delta_{u}, \quad \gamma_{4}+n \delta_{d}
$$

and

$$
\gamma_{2}+n \delta_{u}, \quad \gamma_{3}+n \delta_{d}
$$

As $n \rightarrow \infty$, the towers of states accumulate to the vectors $\delta_{u}, \delta_{d}$, which are vector multiplets for the four-dimensional quivers that decompose the fivedimensional quiver as in Fig. 3a. The mutation operators $\mathbf{m}, \hat{\mathbf{m}}$ are related in a simple way to the time evolution operator:

$$
\mathbf{m}=T_{\mathbb{F}_{0}}^{2}, \quad \hat{\mathbf{m}}=T_{\mathbb{F}_{0}}^{-2} \iota,
$$

where $\iota$ is the inversion. From the perspective of the full automorphism group

$$
\widetilde{W}\left(A_{1}^{(1)}\right) \ltimes D i h_{4},
$$

it is natural to consider also another translation operator

$$
T_{\mathbb{F}_{0}}^{\prime}=(2,3)(1,4) \mu_{2} \mu_{4},
$$

whose action on the charges is

$$
\begin{array}{ll}
\left(T_{\mathbb{F}_{0}}^{\prime}\right)^{n}\left(\gamma_{1}\right)=\gamma_{1}-n\left(\gamma_{1}+\gamma_{4}\right), & \left(T_{\mathbb{F}_{0}}^{\prime}\right)^{n}\left(\gamma_{2}\right)=\gamma_{2}+n\left(\gamma_{2}+\gamma_{3}\right), \\
\left(T_{\mathbb{F}_{0}}^{\prime}\right)^{n}\left(\gamma_{3}\right)=\gamma_{3}-n\left(\gamma_{2}+\gamma_{3}\right), & \left(T_{\mathbb{F}_{0}}^{\prime}\right)^{n}\left(\gamma_{4}\right)=\gamma_{4}+n\left(\gamma_{1}+\gamma_{4}\right) .
\end{array}
$$

This generates different towers of hypermultiplets, which are still organized as two copies of the weakly coupled chamber of four-dimensional super YangMills, with vector multiplets

$$
\delta_{l}=\gamma_{1}+\gamma_{4}, \quad \delta_{r}=\gamma_{2}+\gamma_{3} .
$$

In this way, we find a different infinite chamber, corresponding to the decomposition of the $5 \mathrm{~d}$ BPS quiver as in Fig. $3 \mathrm{~b}$.

We see that considering the natural translation operators associated to the quiver automorphisms builds the correct spectrum for the tame chambers in a simpler way, without the need to consider both left and right mutations. This simplification occurs because we are allowing not just mutations, but also permutations, which are relabelings of the BPS charges. This operation of course has no effect on the resulting spectrum, which is the same as the one emerging from using just the mutation algorithm. However, by using quiver automorphisms, it is possible to construct more elementary dualities of the theory, and the spectrum can be constructed more simply. This plays a crucial rôle in more complicated cases. To illustrate this point, we discuss in the following the cases of local $\mathbb{F}_{1}$ and $\mathrm{dP}_{3}$. 


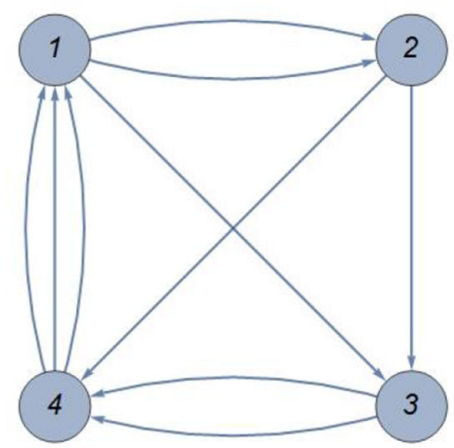

(a) Quiver associated to local $\mathbb{F}_{1}$

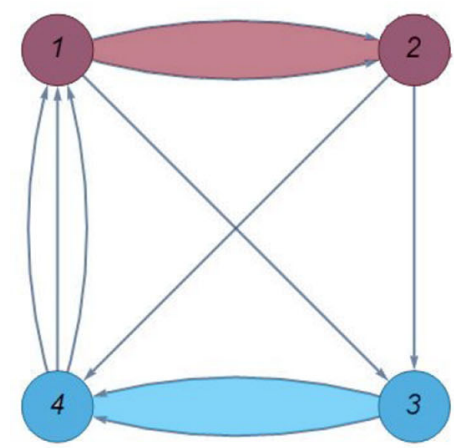

(b) 4d subquivers for local $\mathbb{F}_{1}$

Figure 4. a Quiver associated to local $\mathbb{F}_{1}$. b 4 d subquivers for local $\mathbb{F}_{1}$

\subsection{Super Yang-Mills, $k=1$}

The local $\mathbb{F}_{1}$ quiver is displayed in Fig. 4a. This engineers pure $S U(2)$ SYM with 5 d Chern-Simons level $k=1$. The adjacency matrix is

$$
B=\left(\begin{array}{cccc}
0 & 2 & 1 & -3 \\
-2 & 0 & 1 & 1 \\
-1 & -1 & 0 & 2 \\
3 & -1 & -2 & 0
\end{array}\right)
$$

The translation operator is given by

$$
T_{\mathbb{F}_{1}}=(1324) \mu_{3} .
$$

As far as the spectrum is concerned, this is the same as the local $\mathbb{F}_{0}$ one. The operators $\mathbf{m}, \tilde{\mathbf{m}}$ are easy to build

$$
\mathbf{m} \equiv T_{\mathbb{F}_{1}}^{4}=\left((1324) \mu_{3}\right)^{4}, \quad \hat{\mathbf{m}} \equiv T_{\mathbb{F}_{1}}^{-4} \iota .
$$

and the associated evolution on the vector of charges $\gamma$ is

$$
\begin{aligned}
& T_{\mathbb{F}_{1}}^{2 n}(\gamma)=T_{\mathbb{F}_{0}}^{n}(\gamma)=\left(\gamma_{1}+n \delta_{u} \gamma_{2}-n \delta_{u} \gamma_{3}+n \delta_{d} \gamma_{4}-n \delta_{d}\right), \\
& T_{\mathbb{F}_{1}}^{2 n-1}(\gamma)=\left(\gamma_{3}+n \delta_{d}, \gamma_{4}-n \delta_{d}, \gamma_{2}-n \delta_{u}, \gamma_{1}+n \delta_{u},\right) .
\end{aligned}
$$

We see that even though the introduction of a Chern-Simons level will affect some physical aspects, it does not modify the type of states in the spectrum: again $\delta_{u}, \delta_{d}$ correspond to the vector multiplets of the $4 \mathrm{~d}$ subquivers depicted in Fig. 4b. What changes, however, is the number of tame chambers: because the symmetry group now does not include the $D i h_{4}$ factor - as it is clear by inspection of the quiver - there is not the related chamber.

\section{3. $N_{f}=2, k=0$}

When we include matter the situation is much richer, because we encounter the new feature of multiple commuting flows, each characterizing the spectrum in a different chamber of the moduli space. The relevant quiver is the one of 


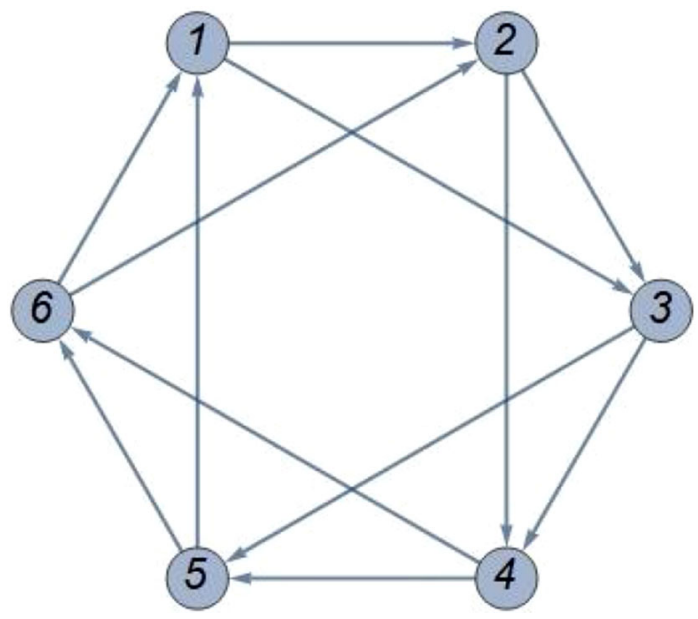

Figure 5. Quiver for $\mathrm{dP}_{3}$

$d P_{3}$, engineering the $S U(2)$ theory with two flavors, depicted in Fig. 5. It has adjacency matrix

$$
B=\left(\begin{array}{cccccc}
0 & 1 & 1 & 0 & -1 & -1 \\
-1 & 0 & 1 & 1 & 0 & -1 \\
-1 & -1 & 0 & 1 & 1 & 0 \\
0 & -1 & -1 & 0 & 1 & 1 \\
1 & 0 & -1 & -1 & 0 & 1 \\
1 & 1 & 0 & -1 & -1 & 0
\end{array}\right)
$$

The extended Weyl group is $\tilde{W}\left(\left(A_{2}+A_{1}\right)^{(1)}\right)$, which is generated by

$$
\begin{array}{lll}
s_{0}=(3,6) \mu_{6} \mu_{3} & s_{1}=(1,4) \mu_{4} \mu_{1}, & s_{2}=(2,5) \mu_{5} \mu_{2}, \\
r_{0}=(4,6) \mu_{2} \mu_{4} \mu_{6} \mu_{2}, & r_{1}=(3,5) \mu_{1} \mu_{3} \mu_{5} \mu_{1}, & \\
\pi=(1,2,3,4,5,6), & \sigma=(1,4)(2,3)(5,6) \iota . &
\end{array}
$$

In this case, there are four commuting evolution operators, given by Weyl translations of $\tilde{W}\left(\left(A_{2}+A_{1}\right)^{(1)}\right)$, acting on the affine root lattice $Q\left(\left(A_{2}+A_{1}\right)^{(1)}\right)$ $[30,34]^{2}$. One has the three operators

$$
T_{1}=s_{0} s_{2} \pi, \quad T_{2}=s_{1} s_{0} \pi, \quad T_{3}=s_{2} s_{1} \pi
$$

satisfying $T_{1} T_{2} T_{3}=1$, and finally

$$
T_{4}=r_{0} \pi^{3} .
$$

Let us consider the flow $T_{1}$ first, given by

$$
T_{1}=s_{0} s_{2} \pi=(3,6) \mu_{6} \mu_{3}(2,5) \mu_{5} \mu_{2}(1,2,3,4,5,6) .
$$

\footnotetext{
${ }^{2}$ For the action on the roots, see Sect. 3.3.
} 


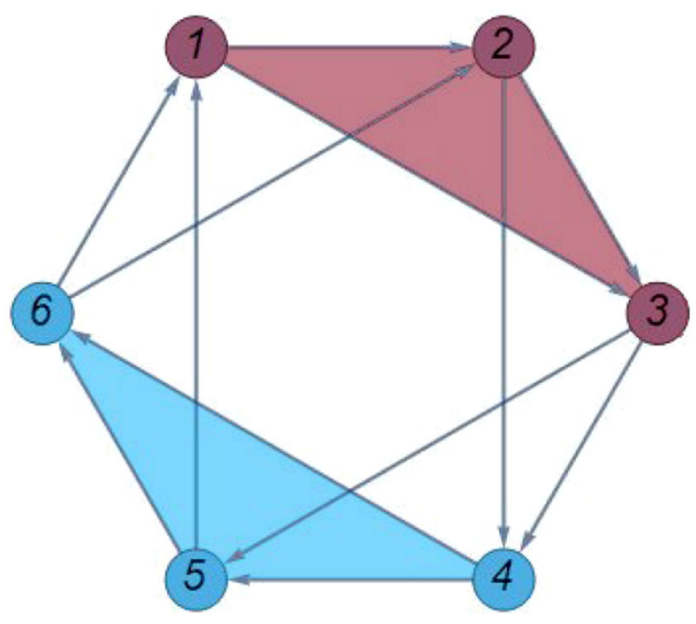

Figure 6. 4d subquivers for local $d P_{3}$, under $T_{1}$

Its action on the BPS charges is the following:

$$
T_{1}^{n}:\left(\begin{array}{c}
\gamma_{1} \\
\gamma_{2} \\
\gamma_{3} \\
\gamma_{4} \\
\gamma_{5} \\
\gamma_{6}
\end{array}\right) \longrightarrow\left(\begin{array}{c}
(n+1) \gamma_{1}+n\left(\gamma_{2}+\gamma_{3}\right), \\
\gamma_{2}, \\
-(n+1)\left(\gamma_{1}+\gamma_{2}\right)-n \gamma_{3}, \\
\gamma_{4}+n\left(\gamma_{4}+\gamma_{5}+\gamma_{6}\right), \\
\gamma_{5}, \\
-(n+1)\left(\gamma_{4}+\gamma_{5}\right)-n \gamma_{6}
\end{array}\right)
$$

We see that $T_{1}$ generates infinite towers of hypermultiplets given by

$$
\begin{array}{ll}
\gamma_{1}+n\left(\gamma_{1}+\gamma_{2}+\gamma_{3}\right), & -(n+1)\left(\gamma_{1}+\gamma_{2}\right)-n \gamma_{3}, \\
\gamma_{4}+n\left(\gamma_{4}+\gamma_{5}+\gamma_{6}\right), & -(n+1)\left(\gamma_{4}+\gamma_{5}\right)-n \gamma_{6} .
\end{array}
$$

These are the BPS states corresponding to two copies of the weakly coupled spectrum for the $N_{f}=1$ theory in four dimensions, and correspond to the decomposition of the $5 \mathrm{~d}$ quiver into two $4 \mathrm{~d}$ subquivers for $N_{f}=1$, as in Fig. 6. One can easily check that the towers of states are mutually local, and as $n \rightarrow \infty$ they accumulate on the rays

$$
\delta_{u}^{(1)}=\gamma_{1}+\gamma_{2}+\gamma_{3}, \quad \delta_{d}^{(1)}=\gamma_{4}+\gamma_{5}+\gamma_{6},
$$

which are indeed the vector multiplets for the $4 \mathrm{~d} N_{f}=1$ subquivers. More precisely, the towers of hypermultiplets above are only half of the towers from $N_{f}=1$ theory. To complete the picture, here we have to consider, like in the pure gauge case, the states constructed from right mutations: these are generated as before by powers of the inverse of the evolution operator, composed with an inversion $\iota$ : 


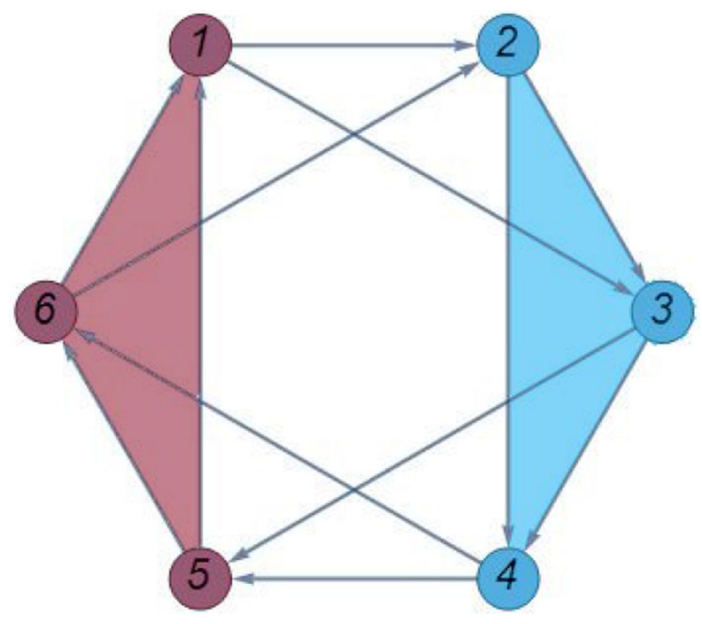

Figure 7. 4d subquivers for local $d P_{3}$, under $T_{2}$

$$
T_{1}^{-n} \iota:\left(\begin{array}{c}
\gamma_{1} \\
\gamma_{2} \\
\gamma_{3} \\
\gamma_{4} \\
\gamma_{5} \\
\gamma_{6}
\end{array}\right) \longrightarrow\left(\begin{array}{c}
-n \gamma_{1}-(n+1)\left(\gamma_{2}+\gamma_{3}\right) \\
\gamma_{2} \\
n \gamma_{3}+(n+1)\left(\gamma_{1}+\gamma_{2}\right) \\
-n \gamma_{4}-(n+1)\left(\gamma_{5}+\gamma_{6}\right) \\
\gamma_{5} \\
(n+1) \gamma_{6}+n\left(\gamma_{5}+\gamma_{4}\right) .
\end{array}\right)
$$

The spectrum of the $N_{f}=1$ theory in four dimensions also includes two quarks that for the subquivers in Fig. 6 are given by $\gamma_{5}, \gamma_{4}+\gamma_{6}, \gamma_{2}, \gamma_{1}+\gamma_{3}$. We see that we recover the quarks $\gamma_{5}, \gamma_{2}$ as the states that are left invariant by $T_{1}$, while the other quarks would be their complementary in the subquiver. We will see below how the remaining quarks can be recovered as the states that are fixed by a different flow.

$T_{2}$ is given by

$$
T_{2}=s_{1} s_{0} \pi=(1,4) \mu_{4} \mu_{1}(3,6) \mu_{6} \mu_{3}(1,2,3,4,5,6),
$$

and acts on the BPS charges as

$$
T_{2}^{n}:\left(\begin{array}{c}
\gamma_{1} \\
\gamma_{2} \\
\gamma_{3} \\
\gamma_{4} \\
\gamma_{5} \\
\gamma_{6}
\end{array}\right) \longrightarrow\left(\begin{array}{c}
-n \gamma_{1}-(n+1)\left(\gamma_{5}+\gamma_{6}\right) \\
(n+1) \gamma_{2}+n\left(\gamma_{3}+\gamma_{4}\right) \\
\gamma_{3} \\
-n\left(\gamma_{2}+\gamma_{3}\right)-n \gamma_{4} \\
(n+1) \gamma_{5}+n\left(\gamma_{1}+\gamma_{6}\right) \\
\gamma_{6} .
\end{array}\right)
$$

Reasoning as before, we find that the spectrum in this chamber is organized in two copies of the $4 \mathrm{~d} N_{f}=1$ weakly coupled chamber, corresponding to the subquiver decomposition in Fig. 7, with vector multiplets

$$
\delta_{2}^{(u)}=\gamma_{1}+\gamma_{5}+\gamma_{6}, \quad \delta_{2}^{(d)}=\gamma_{2}+\gamma_{3}+\gamma_{4} .
$$




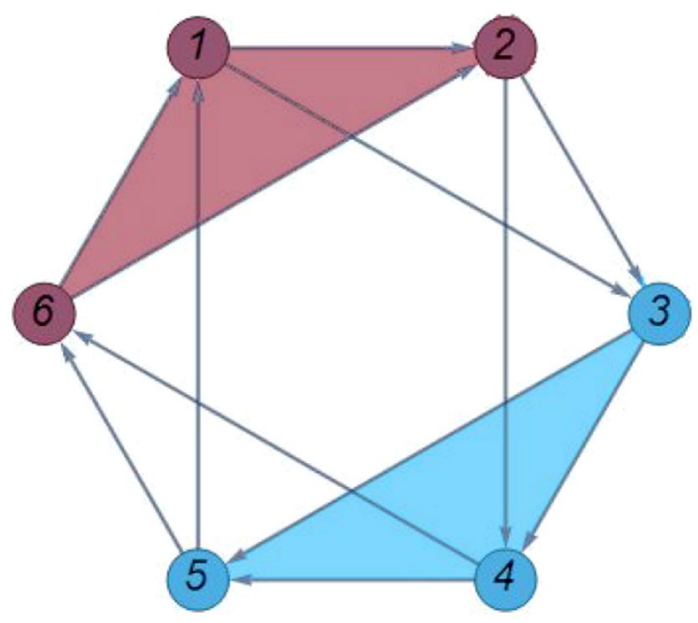

Figure 8. $4 \mathrm{~d}$ subquivers for local $d P_{3}$, under $T_{3}$

Finally, we have

$$
T_{3}=s_{2} s_{1} \pi=(2,5) \mu_{5} \mu_{2}(1,4) \mu_{4} \mu_{1}(1,2,3,4,5,6),
$$

with action

$$
T_{3}^{n}:\left(\begin{array}{c}
\gamma_{1} \\
\gamma_{2} \\
\gamma_{3} \\
\gamma_{4} \\
\gamma_{5} \\
\gamma_{6}
\end{array}\right) \longrightarrow\left(\begin{array}{c}
\gamma_{1} \\
-n \gamma_{2}-(n+1)\left(\gamma_{1}+\gamma_{6}\right) \\
(n+1) \gamma_{3}+n\left(\gamma_{4}+\gamma_{5}\right) \\
\gamma_{4} \\
-n \gamma_{5}-(n+1)\left(\gamma_{3}+\gamma_{4}\right) \\
(n+1) \gamma_{6}+n\left(\gamma_{1}+\gamma_{2}\right)
\end{array}\right)
$$

which gives another chamber organized as two copies of four-dimensional weakly coupled $N_{f}=1$ depicted in Fig. 8, with vector multiplets

$$
\delta_{3}^{(u)}=\gamma_{1}+\gamma_{2}+\gamma_{6}, \quad \delta_{3}^{(d)}=\gamma_{3}+\gamma_{4}+\gamma_{5} .
$$

Before considering the evolution $T_{4}$, let us make a remark. The picture above suggests that there exists a relation between the different flows in terms of permutations of the nodes of the quiver. Indeed, it is possible to check that we have the relations

$$
T_{1}=(3,4,5,6,1,2) T_{2}(5,6,1,2,3,4)=(6,1,2,3,4,5) T_{3}(2,3,4,5,6,1) .
$$

From the point of view of the BPS spectrum, it is now clear that these three flows will generate the same spectrum up to relabeling of states, i.e. they will differ in what we call electric or magnetic in the field theory. Another interesting quiver automorphism is given by

$$
R_{2}=\pi^{2} s_{1}=(3,5,1)(4,2,6)(1,4) \mu_{4} \mu_{1},
$$


which is known as half-translation, because it satisfies $R_{2}^{2}=T_{2}$ (half-translations for $T_{1}, T_{3}$ can be obtained by using equation (2.48)). Under this quiver automorphism, the BPS charges transform as

$$
R_{2}^{2 n+1}:\left(\begin{array}{c}
\gamma_{1} \\
\gamma_{2} \\
\gamma_{3} \\
\gamma_{4} \\
\gamma_{5} \\
\gamma_{6}
\end{array}\right) \longrightarrow\left(\begin{array}{c}
-(n+1) \gamma_{2}-n\left(\gamma_{3}+\gamma_{4}\right) \\
n \gamma_{1}+(n+1)\left(\gamma_{5}+\gamma_{6}\right) \\
\gamma_{1}+\gamma_{5} \\
-(n+1) \gamma_{5}-n\left(\gamma_{1}+\gamma_{6}\right) \\
n \gamma_{4}+(n+1)\left(\gamma_{2}+\gamma_{3}\right) \\
\gamma_{2}+\gamma_{4}
\end{array}\right)
$$

while of course $R_{2}^{2 n}=T_{2}^{n}$. Note that this generates the CPT conjugates of the towers of states as $T_{2}$, while the states that are left fixed by the action of $R_{2}$ are exactly the missing quarks from our analysis of $T_{2}$, so that $R_{2}$ generates the full spectrum of the two copies of $N_{f}=1$ in the subquivers of Fig. 7 .

Finally, the time evolution $T_{4}$ is given by

$$
T_{4}=r_{0} \pi^{3}=(4,6) \mu_{2} \mu_{4} \mu_{6} \mu_{2}(4,5,6,1,2,3),
$$

and acts on the BPS charges as follows:

$$
\begin{aligned}
& T_{4}^{3 n-2}:\left(\begin{array}{l}
\gamma_{1} \\
\gamma_{2} \\
\gamma_{3} \\
\gamma_{4} \\
\gamma_{5} \\
\gamma_{6}
\end{array}\right) \longrightarrow\left(\begin{array}{l}
\gamma_{1}+\left(\gamma_{3}+\gamma_{4}\right)+n \delta \\
\gamma_{2}-\left(\gamma_{1}+\gamma_{2}\right)-n \delta \\
\gamma_{3}+\left(\gamma_{5}+\gamma_{6}\right)+n \delta \\
\gamma_{4}-\left(\gamma_{3}+\gamma_{4}\right)-n \delta \\
\gamma_{5}+\left(\gamma_{1}+\gamma_{2}\right)+n \delta \\
\gamma_{6}-\left(\gamma_{5}+\gamma_{6}\right)-n \delta
\end{array}\right), \\
& T_{4}^{3 n-1}:\left(\begin{array}{l}
\gamma_{1} \\
\gamma_{2} \\
\gamma_{3} \\
\gamma_{4} \\
\gamma_{5} \\
\gamma_{6}
\end{array}\right) \longrightarrow\left(\begin{array}{l}
\gamma_{1}+\left(\gamma_{3}+\gamma_{4}+\gamma_{5}+\gamma_{6}\right)+n \delta \\
\gamma_{2}-\left(\gamma_{1}+\gamma_{2}+\gamma_{3}+\gamma_{4}\right)-n \delta \\
\gamma_{3}+\left(\gamma_{1}+\gamma_{2}+\gamma_{5}+\gamma_{6}\right)+n \delta \\
\gamma_{4}-\left(\gamma_{3}+\gamma_{4}+\gamma_{5}+\gamma_{6}\right)-n \delta \\
\gamma_{5}+\left(\gamma_{1}+\gamma_{2}+\gamma_{3}+\gamma_{4}\right)+n \delta \\
\gamma_{6}-\left(\gamma_{1}+\gamma_{2}+\gamma_{5}+\gamma_{6}\right)-n \delta
\end{array}\right), \\
& T_{4}^{3 n}:\left(\begin{array}{c}
\gamma_{1} \\
\gamma_{2} \\
\gamma_{3} \\
\gamma_{4} \\
\gamma_{5} \\
\gamma_{6}
\end{array}\right) \longrightarrow\left(\begin{array}{c}
\gamma_{1}+n \delta \\
\gamma_{2}-n \delta \\
\gamma_{3}+n \delta \\
\gamma_{4}-n \delta \\
\gamma_{5}+n \delta \\
\gamma_{6}-n \delta
\end{array}\right) .
\end{aligned}
$$

We can recognize in this chamber towers of states that accumulate to the same BPS ray $\delta=\gamma_{1}+\cdots+\gamma_{6}$, representing a multiplet with higher spin $s \geq 1$. In this case, the four-dimensional interpretation is subtler and more interesting, and we postpone it to Sect. 5.3. 


\section{Discrete BPS Quiver Dynamics, Cluster Algebras and q-Painlevé Equations}

The translation operators acting on the BPS quivers we described so far can be regarded as time evolution operators for discrete dynamical systems arising from deautonomization of cluster integrable systems, naturally associated to the geometric engineering of the corresponding five-dimensional gauge theories. This allows to bridge between the BPS quiver description and classical results in the theory of q-Painlevé equations, and actually inspired the reformulation of the BPS quiver analysis that we presented in the previous section. Indeed, the quivers studied in [18] are exactly the 5d BPS quivers appearing in Sect. 3. In this respect, the q-Painlevé flows describe self-dualities (in the sense of section) of the $4 \mathrm{~d}$ Kaluza-Klein theory obtained by reducing the $5 \mathrm{~d}$ gauge theory on $S^{1}$. We now turn to the study of all the examples we considered up to now from this perspective.

\subsection{Cluster Algebras and Quiver Mutations}

Let us first recall the notion of cluster algebra $[35,36]$, as well as the two types of cluster variables that will be used throughout the paper. The ambient field for a cluster algebra $\mathcal{A}$ is a field $\mathcal{F}$ isomorphic to the field of rational functions in $n=\operatorname{rk} \mathcal{A}$ independent variables, with coefficients in $\mathbb{Q P}$, where $\mathbb{P}$ is the tropical semifield. The tropical semifield is defined as follows: starting with the free abelian group $(\mathbb{P}, \cdot)$ with usual multiplication, the operation $\oplus$ is defined in terms of a basis ${ }^{3} \mathbf{u}$ of $\mathbb{P}$

$$
\prod_{j} u_{j}^{a_{j}} \oplus \prod_{j} u_{j}^{b_{j}}=\prod_{j} u_{j}^{\min \left(a_{j}, b_{j}\right)} .
$$

The cluster algebra $\mathcal{A}$ is determined by the choice of an initial seed. This is a triple $(Q, \boldsymbol{\tau}, \mathbf{y})$, where

- $Q$ is a quiver without loops and 2-cycles, with $n$ vertices;

- $\mathbf{y}=\left(y_{1}, \ldots, y_{n}\right)$ is an $n$-tuple of generators of the tropical semifield $(\mathbb{P}, \oplus, \cdot)$ (which in general will not be independent generators, because $\operatorname{dim} \mathbb{P} \leq n)$

- $\boldsymbol{\tau} \equiv\left(\tau_{1}, \ldots, \tau_{n}\right)$ is an n-tuple of elements of $\mathcal{F}$ forming a free generating set: they are algebraically independent over $\mathbb{Q P}$, and $\mathcal{F}=\mathbb{Q P}\left(\tau_{1}, \ldots, \tau_{n}\right)$.

The variables $(\boldsymbol{\tau}, \mathbf{y})$ are called A-cluster variables. We can alternatively define the seed as $(B, \boldsymbol{\tau}, \mathbf{y})$ in terms of the antisymmetric adjacency matrix $B$ of the quiver.

Given these objects, the cluster algebra is the $\mathbb{Z P}$-subalgebra of $\mathcal{F}$ generated recursively by applying mutations to the initial seed. A mutation $\mu_{k}$ is an operation defined by its action on a seed:

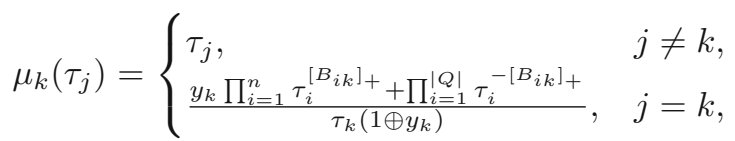

\footnotetext{
${ }^{3}$ We allow for the free abelian group to have dimension less than $n$, as it will typically the case for us.
} 


$$
\begin{aligned}
\mu_{k}\left(y_{j}\right) & = \begin{cases}y_{j}^{-1}, & j=k, \\
y_{j}\left(1 \oplus y_{k}^{\operatorname{sgn} B_{j k}}\right)^{B_{j k}}, & j \neq k,\end{cases} \\
\mu_{k}\left(B_{i j}\right) & = \begin{cases}-B_{i j}, & i=k \text { or } j=k, \\
B_{i j}+\frac{B_{i k}\left|B_{k j}\right|+B_{k j}\left|B_{i k}\right|}{2}, & \end{cases}
\end{aligned}
$$

where we defined $[x]_{+}=\max (x, 0)$. It is clear from the above expression that the coefficients $y_{i}$ represent an exponentiated version of the BPS charges $\gamma_{i}$.

An alternative set of variables are the so-called $\mathrm{X}$-cluster variables $\mathbf{x}=$ $\left(x_{1}, \ldots, x_{n}\right)$, taking values in $\mathcal{F}$. They are defined in terms of the A-variables as

$$
x_{i}=y_{i} \prod_{j=1}^{n} \tau_{j}^{B_{j i}},
$$

and their mutation rules are the same as for coefficients, but with ordinary sum instead of semifield sum:

$$
\mu_{k}\left(x_{j}\right)= \begin{cases}x_{j}^{-1}, & j=k, \\ x_{j}\left(1+x_{k}^{\operatorname{sgn} B_{j k}}\right)^{B_{j k},}, & j \neq k .\end{cases}
$$

The X-cluster variables can be considered as coordinates in the so-called $\mathrm{X}$-cluster variety, which is endowed with a degenerate Poisson bracket, with respect to which the $\mathrm{X}$-cluster variables are log-canonically conjugated:

$$
\left\{x_{i}, x_{j}\right\}=B_{i j} x_{i} x_{j} .
$$

Given a convex Newton polygon $\Delta$ with area $S$, it is possible to construct a quiver with $2 S$ nodes describing a discrete integrable system in the variables $x_{i}[22,37]$. Due to $(3.7)$, in general the Poisson bracket is degenerate, as there is a space of Casimirs equal to $\operatorname{ker}(B)$. For quivers arising in this way, the quantity

$$
q \equiv \prod_{i} x_{i}
$$

is always a Casimir. The system is integrable on the level surface

$$
q=1 \text {. }
$$

The number of independent Hamiltonians is the number of internal points of the Newton polygon. The set of discrete time flows of the integrable system is the group $\mathcal{G}_{Q}$ of quiver automorphisms ${ }^{4}$. We will in fact work with the extended group $\tilde{\mathcal{G}}_{Q}$ that extends $\mathcal{G}_{Q}$ by the inclusion of the inversion operator

\footnotetext{
${ }^{4}$ To be more precise, the discrete time flows are given by a subgroup $\mathcal{G}_{\Delta} \subset \mathcal{G}_{Q}$, of automorphisms preserving the Hamiltonians. These are given by spider moves of the associated dimer model that we are not introducing here. They are very specific mutation sequences that in this work we will rather view as Weyl translations acting on an affine root lattice. For the cases that we will be concerned with in this paper, the two groups coincide and we can forget about the distinction.
} 
$\iota$. This operation reverses all the arrows in the quiver, and acts on the cluster variables as

$$
\iota\left(x_{i}\right)=x_{i}^{-1}, \quad \iota\left(y_{i}\right)=y_{i}^{-1},
$$

while the variables $\tau$ are invariant, consistently with the relation (3.5).

In [18] it was shown that it is possible to obtain q-Painlevé equations by lifting the constraint $q=1$, which amounts to the deautonomization of the system. This is no longer integrable in the Liouville sense, since the discrete Hamiltonians are no longer preserved under the discrete flows. The related equations of motion are well-known q-difference integrable equations of mathematical physics, namely q-Painlevé equations: the time evolution describes in this case a foliation, whose slices are different level surfaces of the original integrable system, see, for example, [38] for such a description of q-Painlevé equations. These equations can be obtained geometrically by studying configurations of blowups of eight points on $\mathbb{P}^{1} \times \mathbb{P}^{1}$, or equivalently by configurations of nine blowups on $\mathbb{P}^{2}$. As in the case of differential Painlevé equations [39], this leads to a classification in terms of the space of their initial conditions, called in this context surface type of the equation, or equivalently by their symmetry groups due to Sakai [5], see Fig. 1. The former are given by an affine algebra, while the latter turns out to be given by the extended Weyl group of another affine algebra, which is the orthogonal complement of the first one in the group of divisors $\operatorname{Pic}(X), X$ being the surface obtained by blowing up points on $\mathbb{P}^{1} \times \mathbb{P}^{1}$.

It was further argued in [18] that the time evolution given by the deautonomization of the cluster integrable system, when written in terms of the cluster A-variables $(\boldsymbol{\tau}, \mathbf{y})$, takes the form of bilinear equations, so that we can identify the variables $\boldsymbol{\tau}$ with tau functions for q-Painlevé equations. However, while the q-Painlevé equations in terms of the $\mathrm{X}$-cluster variables were derived for all the Newton polygons with one internal point in [18], their bilinear form was not obtained, except for the Newton polygon of local $\mathbb{F}_{0}$, corresponding to the q-Painlevé equation of surface type $A_{7}^{(1)^{\prime}}$, and local $\mathbb{F}_{1}$ in [19], corresponding to $A_{7}^{(1)}$ in Sakai's classification.

In the next section, we review these two cases, before turning to the case of $d P_{3}$, which corresponds instead to the surface type $A_{5}^{(1)}$. In fact, this case is much richer, as it admits four commuting discrete flows: we will show that one of these reproduces the bilinear equations considered in $[25,26]$ for q-Painlevé $\mathrm{III}_{1}$.

\subsection{Pure Gauge Theory and q-Painlevé $\mathrm{III}_{3}$}

Let us briefly review how q-Painlevé equations are obtained from the quivers associated to local $\mathbb{F}_{0}$ and local $\mathbb{F}_{1}$, whose Newton polygons are depicted in Fig. 9a, b. These correspond to the pure $S U(2)$ gauge theory with ChernSimons level, respectively, $k=0,1$.

Local $\mathbb{F}_{0}$ :

Let us consider first the cluster algebra associated to the quiver in Fig. 2. This corresponds to local $\mathbb{F}_{0}$. The group $\mathcal{G}_{Q}$ of quiver automorphisms contains 


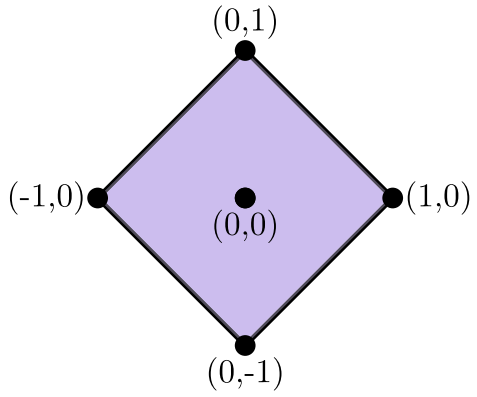

(a) Newton polygon for local $\mathbb{F}_{0}$

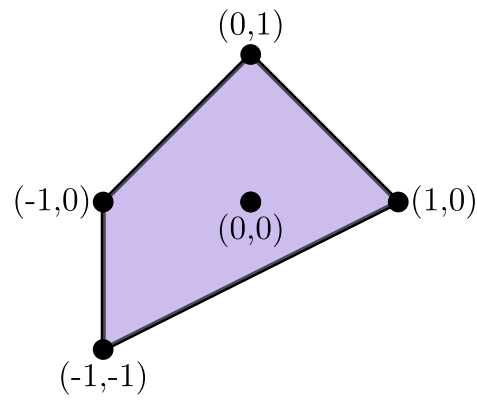

(b) Newton polygon for local $\mathbb{F}_{1}$

Figure 9. a Newton polygon for local $\mathbb{F}_{0}$. b Newton polygon for local $\mathbb{F}_{1}$

the symmetry group of the q-Painlevé equation $\mathrm{qPIII}_{3}$ of surface type $A_{7}^{(1)^{\prime}}$, which is the semidirect product $D i h_{4} \ltimes W\left(A_{1}^{(1)}\right)$. It is generated by

$$
\pi_{1}=(1,3) \iota, \quad \pi_{2}=(4,3,2,1), \quad T_{\mathbb{F}_{0}}=(1,2)(3,4) \mu_{1} \mu_{3} .
$$

The operator $T_{\mathbb{F}_{0}}$ generates the time evolution of the corresponding q-Painlevé equation, and is a Weyl translation on the underlying $A_{1}^{(1)}$ lattice. From the adjacency matrix of the quiver

$$
B=\left(\begin{array}{cccc}
0 & 2 & 0 & -2 \\
-2 & 0 & 2 & 0 \\
0 & -2 & 0 & 2 \\
2 & 0 & -2 & 0
\end{array}\right),
$$

we see that the space of Casimirs of the Poisson bracket (3.7) is two-dimensional. We take the two Casimirs to be

$$
q=\prod_{i} x_{i}=\prod y_{i}, \quad t=x_{2}^{-1} x_{4}^{-1}=y_{2}^{-1} y_{4}^{-1}
$$

Therefore, the tropical semifield has two generators that we take to be the two Casimirs $q$, t. By fixing the initial conditions for the coefficients, consistently with equation (3.13), to be

$$
\mathbf{y}=\left((q t)^{1 / 2}, t^{-1 / 2},(q t)^{1 / 2}, t^{-1 / 2}\right),
$$

one finds that the action of $T_{\mathbb{F}_{0}}$ on the coefficients yields

$$
\bar{q}=q, \quad \bar{t}=q t,
$$

while the tau variables evolve as

$$
\left\{\begin{array} { l } 
{ T _ { \mathbb { F } _ { 0 } } ( \tau _ { 1 } ) = \tau _ { 2 } , } \\
{ T _ { \mathbb { F } _ { 0 } } ( \tau _ { 2 } ) = \frac { \tau _ { 2 } ^ { 2 } + ( q t ) ^ { 1 / 2 } \tau _ { 4 } ^ { 2 } } { \tau _ { 1 } } , } \\
{ T _ { \mathbb { F } _ { 0 } } ( \tau _ { 3 } ) = \tau _ { 4 } , } \\
{ T _ { \mathbb { F } _ { 0 } } ( \tau _ { 4 } ) = \frac { \tau _ { 4 } ^ { 2 } + ( q t ) ^ { 1 / 2 } \tau _ { 2 } ^ { 2 } } { \tau _ { 3 } } }
\end{array} \quad \left\{\begin{array}{l}
T_{\mathbb{F}_{0}}^{-1}\left(\tau_{1}\right)=\frac{\tau_{1}^{2}+t^{1 / 2} \tau_{3}^{2}}{\tau_{2}} \\
T_{\mathbb{F}_{0}}^{-1}\left(\tau_{2}\right)=\tau_{1} \\
T_{\mathbb{F}_{0}}^{-1}\left(\tau_{3}\right)=\frac{\tau_{3}^{2}+t^{1 / 2} \tau_{1}^{2}}{\tau_{4}} \\
T_{\mathbb{F}_{0}}^{-1}\left(\tau_{4}\right)=\tau_{3},
\end{array}\right.\right.
$$


leading to the bilinear equations ${ }^{5}$

$$
\overline{\tau_{1}} \underline{\tau_{1}}=\tau_{1}^{2}+t^{1 / 2} \tau_{3}^{2}, \quad \overline{\tau_{3}} \underline{\tau_{3}}=\tau_{3}^{2}+t^{1 / 2} \tau_{1}^{2} .
$$

The actual q-Painlevé equation is the equation involving the variables $\mathbf{x}$. It takes the form of a system of two first order q-difference equations, or of a single second-order q-difference equation, in terms of log-canonically conjugated variables

$$
F \equiv x_{1}, \quad G=x_{2}^{-1},
$$

that satisfy

$$
\{F, G\}=2 F G \text {. }
$$

Their time evolution can be studied in a completely analogous way by using the mutation rules (3.6) for X-cluster variables, and leads to the q-Painlevé $\mathrm{III}_{3}$ equation

$$
\bar{G} \underline{G}=\left(\frac{G+t}{G+1}\right)^{2} .
$$

Local $\mathbb{F}_{1}$ : We now consider the A-variables associated to the local $\mathbb{F}_{1}$ quiver of Fig. 4a, engineering pure $S U(2)$ SYM with 5 d Chern-Simons level $k=1$. The adjacency matrix is

$$
B=\left(\begin{array}{cccc}
0 & 2 & 1 & -3 \\
-2 & 0 & 1 & 1 \\
-1 & -1 & 0 & 2 \\
3 & -1 & -2 & 0
\end{array}\right)
$$

The corresponding equation is the q-Painlevé equation of surface type $A_{7}^{(1)}$, which is a different q-discretization of the differential Painlevé $\mathrm{III}_{3}$. The time evolution is given by

$$
T_{\mathbb{F}_{1}}=(1324) \mu_{3}
$$

The Casimirs are now

$$
q=\prod_{i} y_{i}, \quad t=y_{1} y_{2}^{-1} y_{3}^{2} .
$$

Consistently with this relation, we choose the following initial conditions for the coefficients:

$$
\mathbf{y}=\left(t^{1 / 2}, t^{1 / 2}, t^{1 / 2}, q t^{-3 / 2}\right) .
$$

This yields the time evolution of the Casimirs

$$
\bar{q}=q, \quad \bar{t}=q^{1 / 2} t,
$$

\footnotetext{
${ }^{5}$ We follow the usual convention that one overline denotes a step forward in discrete time, while one underline denotes a step backwards.
} 
and of the $\tau$-variables

$$
\left\{\begin{array} { l } 
{ T _ { \mathbb { F } _ { 1 } } ( \tau _ { 1 } ) = \tau _ { 4 } } \\
{ T _ { \mathbb { F } _ { 1 } } ( \tau _ { 2 } ) = \frac { \tau _ { 4 } ^ { 2 } + t ^ { 1 / 2 } \tau _ { 1 } \tau _ { 2 } } { \tau _ { 3 } } , } \\
{ T _ { \mathbb { F } _ { 1 } } ( \tau _ { 3 } ) = \tau _ { 1 } , } \\
{ T _ { \mathbb { F } _ { 1 } } ( \tau _ { 4 } ) = \tau _ { 2 } . }
\end{array} \quad \left\{\begin{array}{l}
T_{\mathbb{F}_{1}}^{-1}\left(\tau_{1}\right)=\tau_{3}, \\
T_{\mathbb{F}_{1}}^{-1}\left(\tau_{2}\right)=\tau_{4}, \\
T_{\mathbb{F}_{1}}^{-1}\left(\tau_{3}\right)=\frac{t^{1 / 2} \tau_{1}^{2}+\tau_{3} \tau_{4}}{\tau_{2}}, \\
T_{\mathbb{F}_{1}}^{-1}\left(\tau_{4}\right)=\tau_{1} .
\end{array}\right.\right.
$$

The bilinear equations obtained in this way are

$$
\bar{\tau}_{4} \underline{\tau_{3}}=t^{1 / 2} \tau_{1}^{2}+\tau_{3} \tau_{4}, \quad \quad \bar{\tau}_{2} \underline{\tau_{1}}=\tau_{4}^{2}+t^{1 / 2} \tau_{1} \tau_{2},
$$

which are the same as the equations appearing in [19]

$$
\tau(q t) \tau\left(q^{-1} t\right)=\tau^{2}+t^{1 / 2} \tau\left(q^{1 / 2} t\right) \tau\left(q^{-1 / 2} t\right)
$$

for the single tau function $\tau_{4} \equiv \tau$. The identification is achieved by noting that

$$
\left\{\begin{array}{l}
\tau_{1}=\underline{\tau_{4}}, \\
\tau_{2}=\overline{\overline{\tau_{1}}}, \\
\tau_{3}=\underline{\underline{\tau_{1}}},
\end{array}\right.
$$

so that the first of our bilinear equations becomes

$$
\overline{\bar{\tau}} \underline{\underline{\underline{\tau}}}=\tau^{2}+t^{1 / 2} \bar{\tau} \underline{\tau}
$$

which coincides with (3.28) after using (3.25).

\subsection{Super Yang-Mills with Two Flavors and $\mathrm{qPIII}_{1}$}

We now turn to consider the quiver associated to $d P_{3}$, engineering the $S U(2)$ theory with two flavors, depicted in Fig. 10. It has adjacency matrix

$$
B=\left(\begin{array}{cccccc}
0 & 1 & 1 & 0 & -1 & -1 \\
-1 & 0 & 1 & 1 & 0 & -1 \\
-1 & -1 & 0 & 1 & 1 & 0 \\
0 & -1 & -1 & 0 & 1 & 1 \\
1 & 0 & -1 & -1 & 0 & 1 \\
1 & 1 & 0 & -1 & -1 & 0
\end{array}\right)
$$

and Casimirs

$$
\begin{array}{lll}
a_{0}=\left(y_{3} y_{6}\right)^{-1 / 2}, & a_{1}=\left(y_{1} y_{4}\right)^{-1 / 2}, & a_{2}=\left(y_{2} y_{5}\right)^{-1 / 2}, \\
b_{0}=\left(y_{2} y_{4} y_{6}\right)^{-1 / 2}, & b_{1}=\left(y_{1} y_{3} y_{5}\right)^{-1 / 2},
\end{array}
$$

that satisfy

$$
a_{0} a_{1} a_{2}=b_{0} b_{1}=q^{-1 / 2}, \quad q=y_{1} y_{2} y_{3} y_{4} y_{5} y_{6} .
$$

As already discussed in Sect. 2.3, one has in this case four commuting time evolution operators, given by Weyl translations of $\tilde{W}\left(\left(A_{2}+A_{1}\right)^{(1)}\right)$, which act on the affine root lattice $Q\left(\left(A_{2}+A_{1}\right)^{(1)}\right)$ [30,34]. These time evolutions are not all associated to the same q-Painlevé equation: the three operators

$$
T_{1}=s_{0} s_{2} \pi, \quad T_{2}=s_{1} s_{0} \pi, \quad T_{3}=s_{2} s_{1} \pi
$$



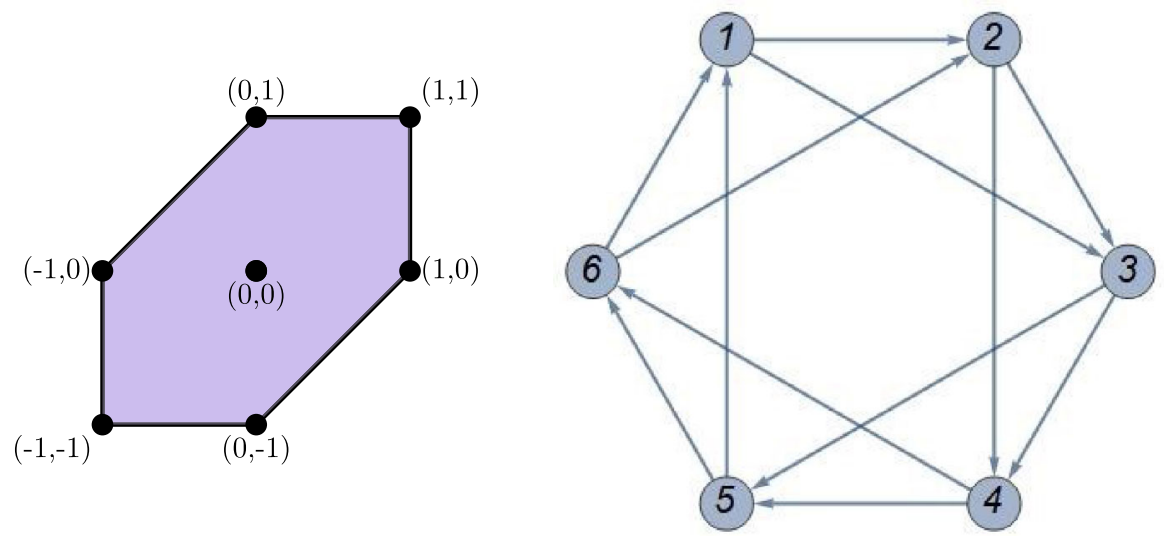

FiguRE 10. Newton polygon and quiver for $\mathrm{dP}_{3}$

give rise to the $\mathrm{q}$-Painlevé equation $\mathrm{qPIII}_{1}$ in the $\mathrm{x}$-variables, and satisfy $T_{1} T_{2} T_{3}=1$. On the other hand, the evolution

$$
T_{4}=r_{0} \pi^{3}
$$

yields q-Painlevé IV.

The time evolution of the Casimirs can be obtained easily from the Xcluster variables: it is

$$
\begin{aligned}
& T_{1}\left(a_{0}, a_{1}, a_{2}, b_{0}, b_{1}, q\right)=\left(q^{1 / 2} a_{0}, q^{-1 / 2} a_{1}, a_{2}, b_{0}, b_{1}, q\right), \\
& T_{2}\left(a_{0}, a_{1}, a_{2}, b_{0}, b_{1}, q\right)=\left(a_{0}, q^{1 / 2} a_{1}, q^{-1 / 2} a_{2}, b_{0}, b_{1}, q\right) \\
& T_{3}\left(a_{0}, a_{1}, a_{2}, b_{0}, b_{1}, q\right)=\left(q^{-1 / 2} a_{0}, a_{1}, q^{1 / 2} a_{2}, b_{0}, b_{1}, q\right), \\
& T_{4}\left(a_{0}, a_{1}, a_{2}, b_{0}, b_{1}, q\right)=\left(a_{0}, a_{1}, a_{2}, q^{-1 / 2} b_{0}, q^{1 / 2} b_{1}, q\right) .
\end{aligned}
$$

This is the counterpart of the fact that $T_{i}$ are Weyl translations acting on the root lattice $Q\left(\left(A_{2}+A_{1}\right)^{(1)}\right)$. If $\alpha_{0}, \alpha_{1}, \alpha_{2}$ are simple roots of $A_{2}^{(1)}$, and $\beta_{0}, \beta_{1}$ are simple roots of $A_{1}^{(1)}$, the action of $T_{i}$ as elements of the affine Weyl group is

$$
\begin{array}{ll}
T_{1}(\boldsymbol{\alpha}, \boldsymbol{\beta})=(\boldsymbol{\alpha}, \boldsymbol{\beta})+(-1,1,0,0,0) \delta, & T_{2}(\boldsymbol{\alpha}, \boldsymbol{\beta})=(\boldsymbol{\alpha}, \boldsymbol{\beta})+(0,-1,1,0,0) \delta \\
T_{3}(\boldsymbol{\alpha}, \boldsymbol{\beta})=(\boldsymbol{\alpha}, \boldsymbol{\beta})+(1,0,-1,0,0) \delta, & T_{4}(\boldsymbol{\alpha}, \boldsymbol{\beta})=(\boldsymbol{\alpha}, \boldsymbol{\beta})+(0,0,0,1,-1) \delta
\end{array}
$$

where $\delta=\alpha_{0}+\alpha_{1}+\alpha_{2}=\beta_{0}+\beta_{1}$ is the null root of $\left(A_{2}+A_{1}\right)^{(1)}$. From each one of these discrete flows, we can obtain bilinear equations for the cluster A-variables $\tau$. Once we choose one of the flows as time, the other flows can be regarded as Bäcklund transformations describing symmetries of the time evolution. 
Let us define the four tropical semifield generators to be $q, t, Q_{1}, Q_{2}$, and the initial condition on the parameters to be

$$
\mathbf{y}=\left(-\frac{1}{Q_{2} t^{1 / 2}}, q^{1 / 4} t^{1 / 2}, Q_{1} q^{1 / 4},-\frac{1}{Q_{1} t^{1 / 2}}, q^{1 / 4} t^{1 / 2}, Q_{2} q^{1 / 4}\right)
$$

which means, in terms of the original parameterization of the Casimirs,

$$
\begin{array}{rlrl}
a_{0}^{2} & =\frac{1}{Q_{1} Q_{2} q^{1 / 2}}, & a_{1}^{2}=Q_{1} Q_{2} t, & a_{2}^{2}=\frac{1}{q^{1 / 2} t}, \\
b_{0}^{2} & =-q^{-1 / 2} \frac{Q_{1}}{Q_{2}}, & b_{1}^{2}=-q^{-1 / 2} \frac{Q_{2}}{Q_{1}} .
\end{array}
$$

We now derive bilinear equations for the discrete flows of this geometry: the time evolution for $T_{1}$ is

$$
\left\{\begin{array} { l } 
{ T _ { 1 } ( \tau _ { 1 } ) = \tau _ { 3 } , } \\
{ T _ { 1 } ( \tau _ { 2 } ) = \frac { \tau _ { 5 } \tau _ { 6 } - Q _ { 2 } t ^ { 1 / 2 } \tau _ { 2 } \tau _ { 3 } } { \tau _ { 1 } } , } \\
{ T _ { 1 } ( \tau _ { 4 } ) = \tau _ { 6 } , } \\
{ T _ { 1 } ( \tau _ { 5 } ) = \frac { \tau _ { 2 } \tau _ { 3 } - Q _ { 1 } t ^ { 1 / 2 } \tau _ { 5 } \tau _ { 6 } } { \tau _ { 4 } } , }
\end{array} \quad \left\{\begin{array}{l}
T_{1}^{-1}\left(\tau_{2}\right)=\frac{\tau_{4} \tau_{5}+Q_{1} q^{1 / 4} \tau_{1} \tau_{2}}{\tau_{3}} \\
T_{1}^{-1}\left(\tau_{3}\right)=\tau_{1}, \\
T_{1}^{-1}\left(\tau_{5}\right)=\frac{\tau_{1} \tau_{2}+Q_{2} q^{1 / 4} \tau_{4} \tau_{5}}{\tau_{6}} \\
T_{1}^{-1}\left(\tau_{6}\right)=\tau_{4} .
\end{array}\right.\right.
$$

The action on the Casimirs is given by (3.37), that means

$$
T_{1}\left(Q_{1}\right)=q^{-1 / 2} Q_{1}, \quad T_{1}\left(Q_{2}\right)=q^{-1 / 2} Q_{2},
$$

We then have

$$
\left\{\begin{array}{l}
\overline{\tau_{2}} \underline{\tau_{3}}=\tau_{5} \tau_{6}-Q_{2} t^{1 / 2} \tau_{2} \tau_{3}, \\
\overline{\tau_{5}} \underline{\tau_{6}}=\tau_{2} \tau_{3}-Q_{1} t^{1 / 2} \tau_{5} \tau_{6}, \\
\underline{\tau_{2}} \tau_{3}=\tau_{5} \underline{\tau_{6}}-Q_{1} q^{1 / 2} \tau_{2} \underline{\tau_{3}}, \\
\underline{\tau_{5}} \tau_{6}=\tau_{2} \underline{\tau_{3}}-Q_{2}^{1 / 2} q^{1 / 2} \tau_{5} \underline{\tau_{6}},
\end{array} \quad \overline{\tau_{i}}=\tau_{i}\left(q^{-1 / 2} Q_{1}, q^{1 / 2} Q_{2}\right) .\right.
$$

The time flow under $T_{2}$ for the A-cluster variables is

$$
\left\{\begin{array}{l}
T_{2}\left(\tau_{2}\right)=\tau_{4}, \\
T_{2}\left(\tau_{3}\right)=\frac{\tau_{3} \tau_{4}+q^{1 / 4} t^{1 / 2} \tau_{1} \tau_{6}}{\tau_{2}}, \\
T_{2}\left(\tau_{5}\right)=\tau_{1}, \\
T_{2}\left(\tau_{6}\right)=\frac{\tau_{1} \tau_{6}+q^{1 / 4} t^{1 / 2} \tau_{3} \tau_{4}}{\tau_{5}},
\end{array}, \quad\left\{\begin{array}{l}
T_{2}^{-1}\left(\tau_{1}\right)=\tau_{5} \\
T_{2}^{-1}\left(\tau_{3}\right)=\frac{-Q_{1} t^{1 / 2} \tau_{5} \tau_{6}+\tau_{2} \tau_{3}}{\tau_{4}} \\
T_{2}^{-1}\left(\tau_{4}\right)=\tau_{2} \\
T_{2}^{-1}\left(\tau_{6}\right)=\frac{-Q_{2} t^{1 / 2} \tau_{2} \tau_{3}+\tau_{5} \tau_{6}}{\tau_{1}}
\end{array}\right.\right.
$$

where the time evolution is given by

$$
T_{2}(t)=q t,
$$

leading to the bilinear equations

$$
\begin{array}{lll}
\overline{\tau_{3}} \tau_{2}=q^{1 / 4} t^{1 / 2} \overline{\tau_{5}} \tau_{6}+\tau_{3} \overline{\tau_{2}}, & \overline{\tau_{6}} \tau_{5}=\overline{\tau_{5}} \tau_{6}+q^{1 / 4} t^{1 / 2} \tau_{3} \overline{\tau_{2}}, \\
\overline{\tau_{2}} \underline{\tau_{3}}=-Q_{1} t^{1 / 2} \tau_{5} \tau_{6}+\tau_{2} \tau_{3}, & \overline{\tau_{5}} \underline{\tau_{6}}=-Q_{2} t^{1 / 2} \tau_{2} \tau_{3}+\tau_{5} \tau_{6} .
\end{array}
$$

In particular, from the flow $T_{2}$ it is possible to reproduce the bilinear equations of [26], thus obtaining an explicit parameterization of the geometric quantities $a_{i}, b_{i}$ coming from the blowup configuration of $\mathbb{P}^{1} \times \mathbb{P}^{1}$ in terms of the Kähler parameters of $\mathrm{dP}_{3}$. 
The discrete flow $T_{3}$ is not independent, being simply given by $T_{3}=$ $T_{1}^{-1} T_{2}^{-1}$, but we write it down for completeness:

$$
\left\{\begin{array} { l } 
{ T _ { 3 } ( \tau _ { 1 } ) = \frac { \tau _ { 1 } \tau _ { 2 } + Q _ { 2 } q ^ { 1 / 4 } \tau _ { 4 } \tau _ { 5 } } { \tau _ { 6 } } , } \\
{ T _ { 3 } ( \tau _ { 3 } ) = \tau _ { 5 } , } \\
{ T _ { 3 } ( \tau _ { 4 } ) = \frac { \tau _ { 4 } \tau _ { 5 } + Q _ { 1 } q ^ { 1 / 4 } \tau _ { 1 } \tau _ { 2 } } { \tau _ { 3 } } , } \\
{ T _ { 3 } ( \tau _ { 6 } ) = \tau _ { 2 } , }
\end{array} \quad \left\{\begin{array}{l}
T_{3}^{-1}\left(\tau_{1}\right)=\frac{\tau_{3} \tau_{4}+q^{1 / 4} t^{1 / 2} \tau_{1} \tau_{6}}{T_{3}^{-1}\left(\tau_{2}\right)=\tau_{6}} \\
T_{3}^{-1}\left(\tau_{4}\right)=\frac{\tau_{1} \tau_{6}+q^{1 / 4} t^{1 / 2} \tau_{3} \tau_{4}}{\tau_{5}} \\
T_{3}^{-1}\left(\tau_{5}\right)=\tau_{3},
\end{array}\right.\right.
$$

leading to the bilinear relations

$$
\left\{\begin{array}{l}
\overline{\tau_{1}} \underline{\tau_{2}}=\tau_{1} \tau_{2}+Q_{2} q^{1 / 4} \tau_{4} \tau_{5}, \\
\overline{\tau_{4}} \underline{\tau_{5}}=\tau_{4} \tau_{5}+Q_{1} q^{1 / 4} \tau_{1} \tau_{2}, \\
\underline{\tau_{1}} \tau_{2}=q^{1 / 4} t^{1 / 2} \tau_{1} \underline{\tau_{2}}+\tau_{4} \underline{\tau_{5}}, \\
\underline{\tau_{4}} .
\end{array}\right.
$$

\subsection{Super Yang-Mills with Two Flavors, q-Painlevé IV and q-Painlevé II}

On top of the previous time evolutions giving rise to $\mathrm{qPIII}_{1}$ equations, there is another the time evolution $T_{4}$ from a further automorphism of the $d P_{3}$ quiver. This gives rise to the qPIV dynamics and has the following action on the Casimirs, dictated by (3.40):

$$
T_{4}\left(Q_{1}\right)=q^{1 / 2} Q_{1}, \quad T_{4}\left(Q_{2}\right)=q^{-1 / 2} Q_{2} .
$$

On the tau variables, this amounts to

$$
\begin{aligned}
& \left\{\begin{array}{l}
T_{4}\left(\tau_{1}\right)=\tau_{4}, \\
T_{4}\left(\tau_{2}\right)=\frac{\tau_{1} \tau_{2} \tau_{6}+\tau_{4} \tau_{5} \tau_{6}-Q_{2} t^{1 / 2} \tau_{2} \tau_{3} \tau_{4}}{\tau_{1} \tau_{3}} \\
T_{4}\left(\tau_{3}\right)=\tau_{6}, \\
T_{4}\left(\tau_{4}\right)=\frac{\tau_{1} \tau_{2} \tau_{6}+\tau_{4} \tau_{5} \tau_{6}+q^{1 / 4} t^{1 / 2} \tau_{2} \tau_{3} \tau_{4}}{\tau_{3} \tau_{5}} \\
T_{4}\left(\tau_{5}\right)=\tau_{2}, \\
T_{4}\left(\tau_{6}\right)=\frac{-Q_{2} t^{1 / 2} \tau_{1} \tau_{2} \tau_{6}+q^{1 / 4} t^{1 / 2} \tau_{4} \tau_{5} \tau_{6}-Q_{2} q^{1 / 4} t \tau_{2} \tau_{3} \tau_{4}}{\tau_{1} \tau_{5}}
\end{array}\right. \\
& \left\{\begin{array}{l}
T_{4}^{-1}\left(\tau_{1}\right)=\frac{\tau_{1} \tau_{2} \tau_{3}+Q_{2} q^{1 / 4} \tau_{3} \tau_{4} \tau_{5}+Q_{2} q^{1 / 2} t^{1 / 2} \tau_{1} \tau_{5} \tau_{6}}{\tau_{2} \tau_{6}}, \\
T_{4}^{-1}\left(\tau_{2}\right)=\tau_{5}, \\
T_{4}^{-1}\left(\tau_{3}\right)=\frac{q^{1 / 4} t^{1 / 2} \tau_{1} \tau_{2} \tau_{3}-Q_{1} t^{1 / 2} \tau_{3} \tau_{4} \tau_{5}-Q_{1} q^{1 / 4} t \tau_{1} \tau_{5} \tau_{6}}{\tau_{2} \tau_{4}}, \\
T_{4}^{-1}\left(\tau_{4}\right)=\tau_{1}, \\
T_{4}^{-1}\left(\tau_{5}\right)=\frac{\tau_{1} \tau_{2} \tau_{3}+Q_{2} q^{1 / 4} \tau_{3} \tau_{4} \tau_{5}-Q_{1} t^{1 / 2} \tau_{1} \tau_{5} \tau_{6}}{\tau_{4} \tau_{6}}, \\
T_{4}^{-1}\left(\tau_{6}\right)=\tau_{3} .
\end{array}\right.
\end{aligned}
$$

At first sight this seems to lead to cubic equations. However, by following the procedure explained in "Appendix B.2", one obtains an equivalent set of bilinear equations 


$$
\left\{\begin{array}{l}
\overline{\tau_{6}} \frac{\tau_{2}}{Q_{1}}-q^{1 / 4} t^{1 / 2} \overline{\tau_{2}} \underline{\tau_{6}}=-t^{1 / 2}\left(Q_{2}+q^{1 / 2} Q_{1}\right) \tau_{2} \tau_{6}, \\
Q_{+}^{1 / 2} q^{1 / 4} \overline{\tau_{6}} \underline{\tau_{4}}+Q_{2} t^{1 / 2} \overline{\tau_{4}} \underline{\tau_{6}}=t^{1 / 2}\left(Q_{2}+q^{1 / 2} Q_{1}\right) \tau_{4} \tau_{6}, \quad, \quad \overline{\tau_{i}}=\tau_{i}\left(q^{1 / 2} Q_{1}, q^{-1 / 2} Q_{2}\right) . \\
\overline{\tau_{4}} \underline{\tau_{2}}-\overline{\tau_{2}} \underline{\tau_{4}}=t^{1 / 2}\left(Q_{2}+q^{1 / 2} Q_{1}\right) \tau_{2} \tau_{4} .
\end{array}\right.
$$

These provide a bilinear form for the qPIV equation, which to our knowledge did not appear in the literature so far.

q-Painlevé II bilinear relations from "half" translations: Given the root lattice $\left(A_{2}+A_{1}\right)^{(1)}$, there exists another time flow that preserves a $\left(A_{1}+A_{1}^{\prime}\right)^{(1)}$ sublattice only $[40,41]$. It corresponds to the q-Painlevé II equation, and it is given by

$$
R_{2}=\pi^{2} s_{1},
$$

which is still an automorphism of the quiver in Fig. 10. Because $R_{2}^{2}=T_{2}$, this flow is also known as half-translation ${ }^{6}$. Its action on the Casimirs is a translational motion (i.e. a good time evolution) only on the locus $a_{0}=q^{-1 / 4}$, i.e. $Q_{+}=1$, on which it acts as

$$
R_{2}\left(a_{1}\right)=q^{1 / 4} a_{1}, \quad R_{2}\left(a_{2}\right)=q^{-1 / 4} a_{2},
$$

corresponding to

$$
R_{2}(t)=q^{1 / 2} t
$$

Its action on the tau-variables reads

$$
\left\{\begin{array} { l } 
{ R _ { 2 } ( \tau _ { 1 } ) = \frac { \tau _ { 3 } \tau _ { 4 } + q ^ { 1 / 4 } t ^ { 1 / 2 } \tau _ { 1 } \tau _ { 6 } } { \tau _ { 2 } } , } \\
{ R _ { 2 } ( \tau _ { 2 } ) = \tau _ { 6 } , } \\
{ R _ { 2 } ( \tau _ { 3 } ) = \tau _ { 1 } , } \\
{ R _ { 2 } ( \tau _ { 4 } ) = \frac { \tau _ { 1 } \tau _ { 6 } + q ^ { 1 / 4 } t ^ { 1 / 2 } \tau _ { 3 } \tau _ { 4 } } { \tau _ { 5 } } , } \\
{ R _ { 2 } ( \tau _ { 5 } ) = \tau _ { 3 } , } \\
{ R _ { 2 } ( \tau _ { 6 } ) = \tau _ { 4 } , }
\end{array} \quad \left\{\begin{array}{l}
R_{2}^{-1}\left(\tau_{1}\right)=\tau_{3}, \\
R_{2}^{-1}\left(\tau_{2}\right)=\frac{\tau_{5} \tau_{6}-Q_{2} t^{1 / 2} \tau_{2} \tau_{3}}{\tau_{1}}, \\
R_{2}^{-1}\left(\tau_{3}\right)=\tau_{5}, \\
R_{2}^{-1}\left(\tau_{4}\right)=\tau_{6}, \\
R_{2}^{-1}\left(\tau_{5}\right)=\frac{\tau_{2} \tau_{3}-Q_{1} t^{1 / 2} \tau_{5} \tau_{6}}{\tau_{4}}, \\
R_{2}^{-1}\left(\tau_{6}\right)=\tau_{2} .
\end{array}\right.\right.
$$

By setting $Q_{1}=Q_{2}^{-1} \equiv Q$, we obtain the bilinear equations

$$
\left\{\begin{array}{l}
\overline{\overline{\tau_{3}}} \underline{\underline{\tau_{6}}}=\tau_{3} \overline{\tau_{6}}+q^{1 / 4} t^{1 / 2} \overline{\tau_{3}} \tau_{6}, \\
\underline{\tau_{3}}=\overline{\tau_{3}} \tau_{6}+q^{1 / 4} t^{1 / 2} \tau_{3} \overline{\tau_{6}}, \\
\overline{\tau_{3}} \underline{\underline{\tau_{6}}}=\underline{\tau_{3}} \tau_{6}-Q^{-1} t^{1 / 2} \tau_{3} \underline{\tau_{6}}, \\
\underline{\underline{\tau_{3}}} \overline{\overline{\tau_{6}}}=\tau_{3} \underline{\tau_{6}}-Q t^{1 / 2} \underline{\tau_{3}} \tau_{6},
\end{array} \quad \overline{\tau_{i}}=\tau_{i}\left(q^{1 / 2} t\right) .\right.
$$

We see that in fact these equations are consistent under the further requirement $Q=-1$. This is because the third and fourth equations are obtained by simply applying $T_{2}^{-1}$ to the first and second one.

According to Sakai's classification (see Fig. 1) and the analysis in [42], this flow correctly points to the Argyres-Douglas theory of $N_{f}=2$ which is, in the four-dimensional limit, governed by the differential PII equation. It would

${ }^{6}$ Other half-translations can be analogously defined from $T_{1}$ and $T_{3}$. 
be interesting to see if the relevant $\tau$-function can be constructed from a $5 \mathrm{~d}$ lift of the matrix model considered in [43].

\subsection{Summary of $\mathrm{dP}_{3}$ Bilinear Equations}

To conclude this section, let us collect here all the flows we found for $d P_{3}$ geometry together with the respective bilinear equations:

$$
\begin{gathered}
T_{1}, \\
Q_{+} \\
=\mathrm{PPIII}_{1},
\end{gathered}
$$

$T_{2}, \mathrm{qPIII}_{1}$,

$\bar{t}=q t$,

$T_{3}, \mathrm{qPIII}_{1}$,

$\bar{t}=t / q, \overline{Q_{+}}=q Q_{+}$

$T_{4}, \mathrm{qPIV}$,

$\overline{Q_{-}}=q Q_{-}$

$$
\begin{array}{r}
R_{2}, \text { qPII, }\left(Q_{1}=Q_{2}^{-}\right. \\
\bar{t}=q^{1 / 2} t,
\end{array}
$$$$
\left\{\begin{array}{l}
\overline{\tau_{2}} \underline{\tau_{3}}=\tau_{5} \tau_{6}-Q_{2} t^{1 / 2} \tau_{2} \tau_{3}, \\
\overline{\tau_{5}} \underline{\tau_{6}}=\tau_{2} \tau_{3}-Q_{1} t^{1 / 2} \tau_{5} \tau_{6}, \\
\underline{\tau_{2}} \tau_{3}=\tau_{5} \underline{\tau_{6}}-Q_{+}^{1 / 2} q^{1 / 2} \tau_{2} \underline{\tau_{3}}, \\
\underline{\tau_{5}} \tau_{6}=\tau_{2} \underline{\tau_{3}}-Q_{+}^{1 / 2} q^{1 / 2} \tau_{5} \underline{\tau_{6}},
\end{array}\right.
$$$$
\left\{\begin{array}{l}
\overline{\tau_{2}} \underline{\tau_{3}}=\tau_{2} \tau_{3}-Q_{1} t^{1 / 2} \tau_{5} \tau_{6}, \\
\overline{\tau_{5}} \underline{\tau_{6}}=\tau_{5} \tau_{6}-Q_{2} t^{1 / 2} \tau_{2} \tau_{3}, \\
\tau_{2} \overline{\tau_{3}}=\overline{\tau_{2}} \tau_{3}+q^{1 / 4} t^{1 / 2} \overline{\tau_{5}} \tau_{6}, \\
\overline{\tau_{5}} \tau_{6}=\tau_{5} \overline{\tau_{6}}+q^{1 / 4} t^{1 / 2} \overline{\tau_{2}} \tau_{3},
\end{array}\right.
$$$$
\left\{\begin{array}{l}
\overline{\tau_{1}} \underline{\tau_{2}}=\tau_{1} \tau_{2}+Q_{+}^{1 / 2} q^{1 / 4} \tau_{4} \tau_{5}, \\
\overline{\tau_{4}} \underline{\tau_{5}}=\tau_{4} \tau_{5}+Q_{+}^{1 / 2} q^{1 / 4} \tau_{1} \tau_{2}, \\
\underline{\tau_{1}} \tau_{2}=q^{1 / 4} t^{1 / 2} \tau_{1} \underline{\tau_{2}}+\tau_{4} \underline{\tau_{5}}, \\
\underline{\tau_{4}} \tau_{5}=q^{1 / 4} t^{1 / 2} \tau_{4} \underline{\tau_{5}}+\tau_{1} \underline{\tau_{2}},
\end{array}\right.
$$

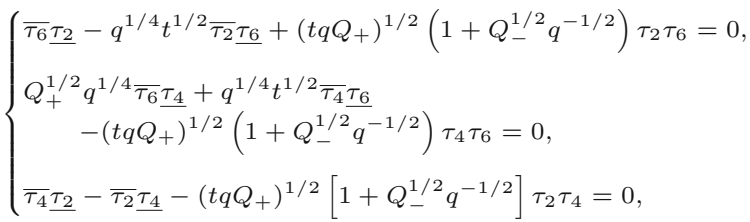

\section{Solutions}

In this Section we discuss how the solutions of the discrete flow of BPS quivers are naturally encoded in topological string partition functions having as a target space the toric Calabi-Yau varieties associated to the relevant Newton polygons. The corresponding geometries are given by rank two vector bundles over punctured Riemann surfaces. Let us recall that the BPS states of the theory are associated to curves on this geometry that locally minimize the string tension. More specifically, hypermultiplets are associated to open curves ending on the branch points of the covering describing the Riemann surface, 
while BPS vector multiplets are associated to closed curves ${ }^{7}$. The BPS states are then described in this setting by open topological string amplitudes with boundaries on those curves. The very structure of the discrete flow suggests to expand the $\tau$ functions as grand canonical partition functions for the relevant brane amplitudes. Specifically, we propose that

$$
\tau_{\left\{m_{i}\right\}}\left(s_{i}, Q_{i}\right)=\sum_{n_{i}} s_{i}^{n_{i}} Z_{t o p}\left(q^{m_{i} n_{i}} Q_{i}\right)
$$

where $q=e^{\hbar}, \hbar=g_{s}$ being the topological string coupling, $Q_{i}$ the Calabi-Yau moduli and $s_{i}$ the fugacities for the branes amplitudes associated to BPS states with intersection numbers $m_{i}$ with the cycles associated to the $Q_{i}$ moduli. These cycles represent a basis associated to the BPS state content of theory in the relevant chamber, the intersection numbers representing the Dirac pairing among them. It is clear from this that the expansion (4.1) for the tau function crucially depends on the BPS chamber. Moreover, distinct flows of the BPS quivers described in the previous sections correspond to bilinear equations in distinct moduli of the Calabi-Yau.

These bilinear equations are in the so-called Hirota form and turn out to be equivalent to convenient combinations of blowup equations [28,44], which consist of many more equations, and suffice to determine recursively the nonperturbative part of the partition function, given the perturbative contribution [45].

In the following, we will mainly focus on the expansion of $\tau$ functions in the electric weakly coupled frame which is suitable to geometrically engineer five-dimensional gauge theories. In this case, the $\tau$ function coincides with the Nekrasov-Okounkov partition function.

\subsection{Local $\mathbb{F}_{0}$ and $q \mathrm{PIII}_{3}$}

We first discuss the pure gauge theory case to gain some perspective. We'll then pass to the richer, and so far less understood, $N_{f}=2$ case. Let us point out here that the solution of $\mathrm{PIII}_{3}$ in the strong coupling expansion was worked out in [9] in terms of the relevant Fredholm determinant (or the matrix model in the cumulants expansion). In [9] also the relevant connection problem was solved. Since we are interested in showing the classical expansion of the cluster variables, here we discuss the different asymptotic expansion in the weak coupling $g_{s} \sim 0$.

In our favorite example, the local $\mathbb{F}_{0}$, the cluster variable $x_{2}=G^{-1}$, where $G$ satisfies (see (3.20))

$$
G(q t) G\left(q^{-1} t\right)=\left(\frac{G(t)+t}{G(t)+1}\right)^{2}
$$

\footnotetext{
${ }^{7}$ Let us notice that in the $5 \mathrm{~d}$ theories on a circle, one finds in general also "wild chambers" with multiplets of higher spin which can be reached via wall-crossing from the "tame" ones. It would be interesting to realize these higher spin multiplets as curves on the spectral geometry.
} 
This can be written in terms of Nekrasov-Okounkov partition functions as

$$
G=i t^{1 / 4} \frac{\tau_{3}}{\tau_{1}}
$$

with

$$
\tau_{1}=\sum_{n \in \mathbb{Z}} s^{n} Z\left(u q^{n}, t\right), \quad \tau_{3}=\sum_{n \in \mathbb{Z}} s^{n+\frac{1}{2}} Z\left(u q^{n+\frac{1}{2}}, t\right),
$$

where $Z$ is the full Nekrasov partition function for which we give explicit formulae in "Appendix A". This case corresponds to the $S U(2)$ pure gauge theory with Chern-Simons level $k=0$, and we set $u_{1}=u_{2}^{-1}=u, q_{1}=q_{2}^{-1}=q$. The bilinear equations

$$
\overline{\tau_{1}} \underline{\tau_{1}}=\tau_{1}^{2}+t^{1 / 2} \tau_{3}^{2}
$$

turn into an infinite set of equations for $Z$ :

$$
\begin{aligned}
& \sum_{n, m} s^{n+m}\left[Z\left(u q^{n}, q t\right) Z\left(u q^{m}, q^{-1} t\right)-Z\left(u q^{n}, t\right) Z\left(u q^{m}, t\right)\right. \\
& \left.\quad-t^{1 / 2} Z\left(u q^{n+1 / 2}, t\right) Z\left(u q^{m-1 / 2}, t\right)\right]=0
\end{aligned}
$$

where the coefficient for each power of $s$ must vanish separately. Of course, most of these equations are redundant, but everything is determined by fixing the asymptotics, i.e. the classical contribution for the partition function. Selecting the term $n+m=1$, for example, we can obtain the following equation for the $t^{0}$ coefficient of $Z$ (i.e. the perturbative contribution):

$$
\frac{Z_{1 \text {-loop }}\left(u q^{-1 / 2}\right) Z_{1 \text {-loop }}\left(u q^{1 / 2}\right)}{Z_{1 \text {-loop }}^{2}}=\frac{1}{u^{2}-1} \frac{1}{u^{-2}-1},
$$

which is the q-difference equation satisfied by $Z_{1 \text {-loop }}$ The term $n+m=0$ allows us to determine the instanton contribution from the perturbative one in the following way:

$$
\begin{aligned}
\sum_{n} Z\left(u q^{n} ; q t\right) Z\left(u q^{-n} ; t / q\right)= & \sum_{n} Z\left(u q^{n} ; t\right) Z\left(u q^{-n} ; t\right) \\
& -t^{1 / 2} \sum_{n} Z\left(u q^{n+1 / 2} ; t\right) Z\left(u q^{-n-1 / 2}\right) .
\end{aligned}
$$

We can express the above equation in terms of $Z_{\text {inst }}$ as

$$
\begin{aligned}
0= & \sum_{n} t^{2 n^{2}} u^{4 n} \prod_{\varepsilon= \pm 1}\left(u^{2 \varepsilon} q^{2 n \varepsilon} ; q, q^{-1}\right)_{\infty} Z_{\text {inst }}\left(u q^{n} ; q t\right) Z_{\text {inst }}\left(u q^{-n} ; t / q\right) \\
& -\sum_{n} t^{2 n^{2}} \prod_{\varepsilon= \pm 1}\left(u^{2 \varepsilon} q^{2 n \varepsilon} ; q, q^{-1}\right)_{\infty} Z_{i n s t}\left(u q^{n} ; t\right) Z_{i n s t}\left(u q^{-n} ; t\right) \\
& +\sum_{n} t^{2(n+1 / 2)^{2}+1 / 2} \prod_{\varepsilon= \pm 1}\left(u^{2 \varepsilon} q^{2(n+1 / 2) \varepsilon} ; q, q^{-1}\right)_{\infty} \\
& Z_{\text {inst }}\left(u q^{n+1 / 2} ; t\right) Z_{\text {inst }}\left(u q^{-(n+1 / 2)} ; t\right) .
\end{aligned}
$$


which gives a recursion relation for the coefficients of the instanton expansion

$$
Z_{\text {inst }}=\sum_{n} t^{n} Z_{n}
$$

For example, the one-instanton term is fully determined just by the perturbative contribution:

$$
\begin{aligned}
Z_{1} & =\frac{2 q}{(q-1)^{2}} \frac{\left(u^{2} q ; q, q^{-1}\right)_{\infty}\left(u^{2} / q ; q, q^{-1}\right)_{\infty}\left(1 / u^{2} q ; q, q^{-1}\right)_{\infty}\left(q / u^{2} ; q, q^{-1}\right)_{\infty}}{\left(u^{2} ; q, q^{-1}\right)_{\infty}^{2}\left(1 / u^{2} ; q, q^{-1}\right)_{\infty}^{2}} \\
& =\frac{2 u^{2} q}{(q-1)^{2}\left(u^{2}-1\right)^{2}} .
\end{aligned}
$$

which of course correctly reproduces the one-instanton Nekrasov partition function.

It is possible to study the autonomous limit of the $\mathrm{X}$-cluster variables by setting

$$
s=e^{\eta / \hbar}, \quad q=e^{\hbar}, \quad u=e^{a},
$$

and sending $\hbar \rightarrow 0$. In this limit one can expand the Nekrasov partition function in the $\Omega$-background parameter as

$$
Z(a, \hbar, t)=\exp \left\{\frac{1}{\hbar^{2}} \sum_{n=0}^{\infty} \hbar^{2 n} F_{n}(a, t)\right\} .
$$

The behavior of the Fourier series in this limit is determined by a discrete version of the saddle point approximation [46]. Let us call $n_{*}$ the saddle point for $n$ : for given $a, \eta$ the saddle point condition

$$
\eta \simeq-F_{0}^{\prime}\left(a+\hbar n_{*}\right)+\mathcal{O}\left(\hbar^{2}\right)
$$

can be satisfied only approximately. More precisely, denote by $a_{*}$ the "true" saddle value, given by the condition

$$
\eta=-F_{0}^{\prime}\left(a_{*}\right)+\mathcal{O}\left(\hbar^{2}\right) .
$$

In general $a+\hbar n_{*}$ will be able to approximate this value only up to $\hbar$ corrections. We write this as

$$
a+\hbar n_{*}=a_{*}+\hbar x,
$$

where the variable $x \sim O(1)$ measures the offset between the true saddle and the approximate one. We review in "Appendix C" the computation of the leading and subleading order in the $\hbar \rightarrow 0$ limit for the dual partition functions, first performed in [19]. The result is that

$$
\begin{aligned}
& \tau_{1}=e^{\frac{1}{\hbar^{2}}\left[\eta\left(a_{*}-a\right)+F_{0}\left(a_{*}, t\right)+F_{1}\left(a_{*}, t\right)+i \pi \tau_{S W} x^{2}+\mathcal{O}(\hbar)\right]} \vartheta_{3}\left(\tau_{S W} x \mid \tau_{S W}\right), \\
& \tau_{3}=e^{\frac{1}{\hbar^{2}}\left[\eta\left(a_{*}-a\right)+F_{0}\left(a_{*}, t\right)+F_{1}\left(a_{*}, t\right)+i \pi \tau_{S W} x^{2}+\mathcal{O}(\hbar)\right]} \vartheta_{2}\left(\tau_{S W} x \mid \tau_{S W}\right) .
\end{aligned}
$$

When we consider the q-Painlevé transcendent, given by the cluster variable $x_{2}^{-1}$, the pre-factor simplifies, so that it is given by a ratio of theta functions:

$$
G=x_{2}^{-1}=i t^{1 / 4} \frac{\tau_{3}}{\tau_{1}}=i t^{1 / 4} \frac{\vartheta_{2}\left(\tau_{S W} x \mid \tau_{S W}\right)}{\vartheta_{3}\left(\tau_{S W} x \mid \tau_{S W}\right)} e^{\mathcal{O}(\hbar)}
$$


The above analysis can be easily extended to the local $\mathbb{F}_{1}$ case by making use of the results in [18]. We do not repeat it here.

\section{2. q-Painlevé III $_{1}$ and Nekrasov Functions}

In the case of the gauge theory with matter, let us first focus on the bilinear equations generated by the translation $T_{2}$. As computed in the previous section, these are

$$
\begin{array}{lll}
\overline{\tau_{3}} \tau_{2}=q^{1 / 4} t^{1 / 2} \overline{\tau_{5}} \tau_{6}+\tau_{3} \overline{\tau_{2}}, & \overline{\tau_{6}} \tau_{5}=\overline{\tau_{5}} \tau_{6}+q^{1 / 4} t^{1 / 2} \tau_{3} \overline{\tau_{2}}, \\
\overline{\tau_{2}} \underline{\tau_{3}}=-Q_{1} t^{1 / 2} \tau_{5} \tau_{6}+\tau_{2} \tau_{3}, & \overline{\tau_{5}} \underline{\tau_{6}}=-Q_{2} t^{1 / 2} \tau_{2} \tau_{3}+\tau_{5} \tau_{6} .
\end{array}
$$

Let us crucially note that these coincide with the bilinear equations studied in [26], eqs (4.5-4.8) after relabeling

$$
\left(\tau_{2}, \tau_{3}\right) \rightarrow\left(\tau_{1}, \tau_{2}\right), \quad\left(\tau_{5}, \tau_{6}\right) \rightarrow\left(\tau_{3}, \tau_{4}\right),
$$

and the identification

$$
Q_{1}=q^{-\theta_{1}}, \quad Q_{2}=q^{-\theta_{2}} .
$$

In the gauge theory, $Q_{1}, Q_{2}$ parameterize the masses of the fundamental hypermultiplets through $\hbar \theta_{i}=m_{i}$, where $\hbar$ is the self-dual $\Omega$-background parameter, while $t$ is the instanton counting parameter. In terms of these, the time evolution is

$$
T_{2}(t)=q t,
$$

so that the discrete time evolution shifts the gauge coupling while the masses stay constant. We can therefore write the bilinear equations as q-difference equations

$$
\left\{\begin{array}{l}
\tau_{2}(q t) \tau_{3}\left(q^{-1} t\right)=\tau_{2}(t) \tau_{3}(t)-Q_{1} t^{1 / 2} \tau_{5}(t) \tau_{6}(t), \\
\tau_{5}(q t) \tau_{6}\left(q^{-1} t\right)=\tau_{5}(t) \tau_{6}(t)-Q_{2} t^{1 / 2} \tau_{2}(t) \tau_{3}(t), \\
\tau_{2}(t) \tau_{3}(q t)=\tau_{2}(q t) \tau_{3}(t)+q^{1 / 4} t^{1 / 2} \tau_{5}(q t) \tau_{6}(t), \\
\tau_{5}(q t) \tau_{6}(t)=\tau_{5}(t) \tau_{6}(q t)+q^{1 / 4} t^{1 / 2} \tau_{2}(q t) \tau_{3}(t) .
\end{array}\right.
$$

It was shown in [26] that the above bilinear equations are solved in terms of the dual partition function for $S U(2)$ SYM with two fundamental flavors. More precisely, in that paper it was shown that if we define

$$
\begin{aligned}
& Z_{0}^{D} \equiv \sum_{n} s^{n} Z\left(Q_{1}, Q_{2}, u q^{n}, t\right), \\
& Z_{1 / 2}^{D}=\sum_{n} s^{n} Z\left(Q_{1}, Q_{2}, u q^{n+1 / 2}, t\right)=Z_{0}^{D}\left(u q^{1 / 2}\right),
\end{aligned}
$$

where $Z$ is the Nekrasov partition function for the $N_{f}=2$ theory, the $\tau$ functions solving (4.25) can be written as

$$
\begin{array}{ll}
\tau_{2}=Z_{0}^{D}\left(Q_{1} q^{1 / 2}, Q_{2}, t q^{-1 / 2}\right), & \tau_{3}=Z_{0}^{D}\left(Q_{1} q^{-1 / 2}, Q_{2}, t q^{1 / 2}\right), \\
\tau_{5}=Z_{1 / 2}^{D}\left(Q_{1}, Q_{2} q^{1 / 2}, t q^{-1 / 2}\right), & \tau_{6}=Z_{1 / 2}^{D}\left(Q_{1}, Q_{2} q^{-1 / 2}, t q^{1 / 2}\right) .
\end{array}
$$

By using also $\tau_{4}=T_{2}\left(\tau_{2}\right), \tau_{1}=T_{2}\left(\tau_{5}\right)$, we can add to these

$$
\tau_{1}=Z_{1 / 2}^{D}\left(Q_{1}, Q_{2} q^{1 / 2}, t q^{1 / 2}\right), \quad \tau_{4}=Z_{0}^{D}\left(Q_{1} q^{1 / 2}, Q_{2}, t q^{1 / 2}\right)
$$


Working in the same way as in Sect. 4.1, one can arrive at bilinear equations for Nekrasov functions, but differently from what happened in that simpler case, now one equation does not suffice to determine the non-perturbative contribution from the perturbative one: we have to use both the first and third equations of (4.25). The first equation takes the form

$$
\begin{aligned}
& \sum_{n} t^{2 n^{2}} u^{2 n} Z_{1-1} Z_{\text {inst }}\left(Q_{1} q^{1 / 2}, u q^{n} ; t q^{1 / 2}\right) Z_{1-1} Z_{\text {inst }}\left(Q_{1} q^{-1 / 2}, u q^{-n}, t q^{-1 / 2}\right) \\
& =\sum_{n} t^{2 n^{2}} u^{-2 n} Z_{1-1} Z_{\text {inst }}\left(Q_{1} q^{1 / 2}, u q^{n} ; t q^{-1 / 2}\right) Z_{1-1} Z_{\text {inst }}\left(Q_{1} q^{-1 / 2}, u q^{-n}, t q^{1 / 2}\right) \\
& \quad-t^{1 / 2} Q_{1} \sum_{r \in \mathbb{Z}+1 / 2} t^{2 r^{2}} u^{-2 r} Z_{1-1} Z_{\text {inst }}\left(Q_{2} q^{1 / 2}, u q^{r}, t q^{-1 / 2}\right) Z_{1-1} \\
& \quad Z_{\text {inst }}\left(Q_{2} q^{-1 / 2}, u q^{-r}, t q^{1 / 2}\right) .
\end{aligned}
$$

This leads to the following equation on the one-instanton contribution:

$$
\begin{aligned}
& q^{-1 / 2}(1-q)\left[Z_{1}\left(Q_{1} q^{-1 / 2}\right)-Z_{1}\left(Q_{1} q^{1 / 2}\right)\right] \\
& =\frac{u}{Q_{1}} \frac{Z_{1-1}\left(Q_{2} q^{-1 / 2}, u q^{1 / 2}\right) Z_{1-1}\left(Q_{2} q^{1 / 2}, u q^{-1 / 2}\right)}{Z_{1-1}\left(Q_{1} q^{1 / 2}\right) Z_{1-1}\left(Q_{1} q^{-1 / 2}\right)} \\
& \quad+\frac{1}{Q_{1} u} \frac{Z_{1-1}\left(Q_{2} q^{-1 / 2}, u q^{-1 / 2}\right) Z_{1-1}\left(Q_{2} q^{1 / 2}, u q^{1 / 2}\right)}{Z_{1-1}\left(Q_{1} q^{1 / 2}\right) Z_{1-1}\left(Q_{1} q^{-1 / 2}\right)} .
\end{aligned}
$$

Differently from what happened in the pure gauge case, one equation is no longer enough to determine the partition function, because we get two occurrences of the function with different shifts on the mass parameter $Q_{1}$. We have to use the third equation of (4.25) that leads to

$$
\begin{aligned}
& \sum_{n} t^{2 n^{2}} u^{4 n} q^{n^{2}} Z_{1-1} Z_{\text {inst }}\left(Q_{1} q^{1 / 2}, u q^{n} ; t q^{-1 / 2}\right) Z_{1-1} Z_{\text {inst }}\left(Q_{1} q^{-1 / 2}, u q^{-n} ; t q^{3 / 2}\right) \\
& =\sum_{n} t^{2 n^{2}} q^{n^{2}} Z_{1-1} Z_{\text {inst }}\left(Q_{1} q^{1 / 2}, u q^{n} ; t q^{1 / 2}\right) Z_{1-1} Z_{\text {inst }}\left(Q_{1} q^{-1 / 2}, u q^{-n} ; t q^{1 / 2}\right) \\
& \quad+q^{1 / 4} t^{1 / 2} \sum_{r} t^{2 r^{2}} q^{r^{2}} Z_{1-1} Z_{\text {inst }}\left(Q_{2} q^{1 / 2}, u q^{r}, t q^{1 / 2}\right) Z_{1-1} Z_{\text {inst }}\left(Q_{2} q^{-1 / 2}, u q^{-r} ; t q^{1 / 2}\right)
\end{aligned}
$$

that gives an equation for the one-instanton contribution

$$
\begin{aligned}
(1-q) & {\left[q Z_{1}\left(Q q^{1 / 2}\right)-Z_{1}\left(Q_{1} q^{-1 / 2}\right)\right] } \\
= & q \frac{Z_{1-1}\left(u q^{-1 / 2}, Q_{2} q^{1 / 2}\right) Z_{1-1}\left(u q^{1 / 2}, Q_{2} q^{-1 / 2}\right)}{Z_{1-1}\left(Q_{1} q^{-1 / 2}\right) Z_{1-1}\left(Q_{1} q^{1 / 2}\right)} \\
& +q \frac{Z_{1-1}\left(u q^{-1 / 2}, Q_{2} q^{-1 / 2}\right) Z_{1-1}\left(u q^{1 / 2}, Q_{2} q^{1 / 2}\right)}{Z_{1-1}\left(Q_{1} q^{-1 / 2}\right) Z_{1-1}\left(Q_{1} q^{1 / 2}\right)} .
\end{aligned}
$$

Putting the two equations together, and using the identities (A.10) and (A.11), we obtain the correct one-instanton contribution

$$
Z_{1}=\frac{q u^{2}}{\left(1-u^{2}\right)^{2}(1-q)^{2}}\left[\left(1-\frac{u}{Q_{1}}\right)\left(1-\frac{u}{Q_{2}}\right)+\left(1-\frac{1}{u Q_{1}}\right)\left(1-\frac{1}{u Q_{2}}\right)\right],
$$


matching the one computed by instanton counting. One can go on and compute the higher instanton contributions in an analogous way. These two equations are enough to determine the non-perturbative contribution order by order in $t$, starting from the knowledge of the perturbative contribution, which is the $t^{0}$ term.

Let us finally note that all these bilinear equations could be written as lattice equations on $Q\left(\left(A_{2}+A_{1}\right)^{(1)}\right)$ by noting that all the various tau functions can be obtained starting from a single one, let us say $\tau_{1}$, since we have

$$
\begin{aligned}
& \tau_{2}=T_{2}^{-1}\left(T_{4}\left(\tau_{1}\right)\right), \quad \tau_{3}=T_{1}\left(\tau_{1}\right), \quad \tau_{4}=T_{4}\left(\tau_{1}\right), \\
& \tau_{5}=T_{2}^{-1}\left(\tau_{1}\right), \quad \tau_{6}=T_{4}^{-1}\left(T_{1}\left(\tau_{1}\right)\right),
\end{aligned}
$$

so that it is possible to introduce, following [34], the tau lattice

$$
\begin{aligned}
\tau_{N}^{k, m} & \equiv T_{1}^{k} T_{2}^{m} T_{4}^{N}\left(\tau_{1}\right) \\
& =Z_{0}^{D}\left(q^{-\frac{N+k}{2}} Q_{1}, q^{\frac{N-k+1}{2}} Q_{2}, q^{\frac{k+N}{2}} u, q^{m} t\right) .
\end{aligned}
$$

In this notation the original tau-variables can be denoted by

$$
\begin{array}{lll}
\tau_{1} \equiv \tau_{0}^{0,0}, & \tau_{2} \equiv \tau_{1}^{0,-1}, & \tau_{3} \equiv \tau_{0}^{1,0}, \\
\tau_{4} \equiv \tau_{1}^{0,0}, & \tau_{5} \equiv \tau_{0}^{0,-1}, & \tau_{6} \equiv \tau_{-1}^{1,0},
\end{array}
$$

and the time flows are integer shifts of the indices of the tau function (4.37). However, not all the flows are compatible with the instanton expansion: from (3.47) and (3.55) we see that the natural expansion parameter for the solution of the $T_{1}$ and $T_{4}$ flows are, respectively, $Q_{1} Q_{2}$ and $Q_{2} / Q_{1}$.

In fact, the usual Nekrasov expansion, as defined in "Appendix A" by a converging expansion in $t$, can only solve the equations for $T_{2}$, which have $t$ as time parameter: this is because if we try to solve the other equations iteratively by starting with the perturbative contribution as defined in equation (A.7), there is no region in parameter space where all the multiple q-Pochhammer functions with shifted arguments entering the bilinear equations have converging expressions simultaneously. To find a solution, one should find an analogue of the perturbative partition function (A.7) which is of order zero, not in $t$, but rather in the appropriate time parameter, solving the order zero of the bilinear equations. This indeed corresponds to an expansion of the topological string partition function (4.1) in the corresponding patch in the moduli space in the Topological Vertex formalism [3].

A preliminary analysis shows that, on top of the evolution in the mass parameters, comparing with the solution in terms of Nekrasov functions, we see that consistency requires also that

$$
T_{1}(u)=q^{1 / 2} u \quad \text { and } \quad T_{4}(u)=q^{1 / 2} u .
$$

To see why this must hold, one has to consider tau functions related by time evolutions of the flows $T_{1}, T_{4}$. For example, the action on the flow $T_{1}$ on the 
solutions $\tau_{1}, \tau_{3}$ (the same considerations would hold by considering the other tau functions):

$$
\tau_{3}=T_{1}\left(\tau_{1}\right), \quad \tau_{1}=Z_{0}^{D}\left(Q_{1}, Q_{2} q^{1 / 2}, u q^{1 / 2}, t q^{1 / 2}\right), \quad \tau_{3}=Z_{0}^{D}\left(Q_{1} q^{-1 / 2}, Q_{2}, u, t q^{1 / 2}\right) .
$$

Equation (4.40) is consistent with the interpretation of the flows $T_{1}, T_{3}, T_{4}$ as the Bäcklund transformations of $T_{2}$.

\section{Degeneration of Cluster Algebras and Four-Dimensional Gauge Theory}

In the previous sections we saw how the cluster algebra structure yields the q-difference equations satisfied by the partition function of the theory, and produces the spectrum of the theory in a weakly coupled chamber by a systematic application of the generalized mutation algorithm. Further, it was observed in [17] that the BPS quivers describing the purely four-dimensional theory (with all KK modes decoupled) are contained in the five-dimensional one as subquivers with two fewer nodes: roughly, one of the additional nodes is the five-dimensional instanton monopole, while the other corresponds to the KK tower of states. From the point of view of cluster integrable systems and q-Painlevé equations, this was already realized in [18]. Graphically, to go from the $5 \mathrm{~d}$ theory to the $4 \mathrm{~d}$ one, one "pops" two nodes of the quiver.

We now show how it is possible to explicitly implement the operation of deleting the two nodes that brings the five-dimensional quiver to the fourdimensional one, at the level of the full cluster algebra, so that we recover the four-dimensional description of the BPS states. From the gauge theory point of view, the four-dimensional limit $\mathbb{R}^{4} \times S_{R}^{1} \rightarrow \mathbb{R}^{4}$ is obtained by taking the radius of the five-dimensional circle $R \rightarrow 0$. More precisely, one has to scale the Kähler parameters in such a way that the KK modes and instanton particles decouple from the BPS spectrum. This limit is usually achieved by implementing the geometric engineering limit $[47,48]$, and takes the form

$$
t=\left(\frac{R \Lambda}{2}\right)^{4-N_{f}}, \quad q=e^{-R g_{s}}, \quad u=e^{-2 a R}, \quad R \rightarrow 0 .
$$

We see that this limit amounts to sending

$$
q \rightarrow 1, \quad t \rightarrow 0, \quad \frac{t}{(\log q)^{4-N_{f}}} \text { finite. }
$$

Because this limit involves

$$
\log q=\log \left(\prod_{i} x_{i}\right)
$$

while the other Casimir is still given by a product of cluster variables, we can already see that it is unlikely for this limit to be able to reproduce cluster 
algebra transformations. Another way to see this is the case, consider the relation between $\mathrm{X}$ - and $\mathrm{A}$-cluster variables for the case of local $\mathbb{F}_{0}$ :

$$
\begin{aligned}
x_{1} & =\left(\frac{\tau_{4}}{\tau_{2}}\right)^{2}(q t)^{1 / 2}, & x_{2} & =\left(\frac{\tau_{1}}{\tau_{3}}\right)^{2} t^{-1 / 2}, \\
x_{3} & =\left(\frac{\tau_{2}}{\tau_{4}}\right)^{2}(q t)^{1 / 2}, & x_{4} & =\left(\frac{\tau_{3}}{\tau_{1}}\right)^{2} t^{-1 / 2} .
\end{aligned}
$$

Because of this, if we implement the limit $t \rightarrow 0, q \rightarrow 1$ by using the geometric engineering prescription, the tau functions, which are given in terms of five-dimensional Nekrasov partition function, will simply go to their fourdimensional limit. Then, no X-cluster variable has an interesting limit. In fact, this is instead the continuous limit of the corresponding Painlevé equation, in which the q-discrete equations become differential equations (see, for example, [21] for the explicit implementation of the limit on the 5d Nekrasov functions).

We will now show how to instead implement the limit $q \rightarrow 1, t \rightarrow 0$ for the cases we considered in this paper: local $\mathbb{F}_{0}, \mathbb{F}_{1}$, and $\mathrm{dP}_{3}$ (respectively, $5 \mathrm{~d}$ pure gauge theory without and with Chern-Simons term, and the theory with $N_{f}=2$ hypermultiplets), in such a way that the cluster algebra structure of the quiver is preserved: in particular we will see that:

- The mutations of the five-dimensional quiver degenerate to those of the four-dimensional one in terms of the reduced set of variables;

- The q-Painlevé time flows (or a sub-flow, in the case of $\mathbb{F}_{1}$ ), which were given by automorphisms of the five-dimensional quivers, degenerate to appropriate sequences of mutations and permutations which are automorphisms of the four-dimensional ones.

Of course, the recipe taken to implement these limit is quite general, and we have no reason to expect it not to work for the other cases. We will implement the limit on the X-cluster variables, because they carry no ambiguity related to the choice of coefficient/extended adjacency matrix. The four-dimensional cluster A-variables can then be obtained from the X-cluster variables using the adjacency matrix as usual. However, because we are implementing this limit on the $\mathrm{X}$-cluster variables, we do not have an explicit expression in terms of Nekrasov functions for the limiting system.

\subsection{From local $\mathbb{F}_{0}$ to the Kronecker Quiver}

Recall the expression for the Casimirs in terms of the cluster variables:

$$
t=x_{2}^{-1} x_{4}^{-1}=x_{1} x_{3} / q, \quad q=x_{1} x_{2} x_{3} x_{4} .
$$

Let us say that we want to decouple the nodes 3,4 on the corresponding quiver, so that we remain with the Kronecker quiver with nodes 1,2 (the red quiver in Fig. 3a). We need then to implement the limit

$$
t \rightarrow 0, \quad q \rightarrow 1, \quad x_{1}, x_{2} \text { finite }
$$


which, as we argued above, is different from the geometric engineering limit. We then have to take

$$
x_{3}=q t / x_{1} \rightarrow 0, \quad x_{4}=\frac{1}{x_{2} t} \rightarrow \infty, \quad x_{3} x_{4} \text { finite. }
$$

We are interested in the expressions for the mutations at the remaining nodes after decoupling, as well as for the q-Painlevé translation. For the mutations, this case is very simple: the limit takes the form

$$
\begin{aligned}
& \mu_{1}(\mathbf{x})=\left(x_{1}^{-1}, \frac{x_{2}}{\left(1+x_{1}^{-1}\right)^{2}}, x_{3},\left(1+x_{1}\right)^{2} x_{4}\right) \rightarrow\left(x_{1}^{-1}, \frac{x_{2}}{\left(1+x_{1}^{-1}\right)^{2}}, 0, \infty\right), \\
& \mu_{2}(\mathbf{x})=\left(x_{1}\left(1+x_{2}\right)^{2}, x_{2}^{-1}, \frac{x_{3}}{\left(1+x_{2}^{-1}\right)^{2}}, x_{4}\right) \rightarrow\left(x_{1}\left(1+x_{2}\right)^{2}, x_{2}^{-1}, 0, \infty\right) .
\end{aligned}
$$

We see that the mutations for $x_{1}, x_{2}$ do not involve the variables $x_{3}, x_{4}$, so that no limit is actually necessary, and in fact they are already in the form of mutations for the Kronecker subquiver. Further, these mutations preserve the limiting value of $x_{3}, x_{4}$. In fact, the choice of the subquiver is completely arbitrary: by this limiting procedure we can consider any of the Kronecker subquivers of the quiver in Fig. 2. The limit is less trivial on the q-Painlevé flow:

$$
T_{\mathbb{F}_{0}}(\mathbf{x})=\left(x_{2} \frac{\left(1+x_{3}\right)^{2}}{\left(1+x_{1}^{-1}\right)^{2}}, x_{1}^{-1}, x_{4} \frac{\left(1+x_{1}\right)^{2}}{\left(1+x_{3}^{-1}\right)^{2}}, x_{3}^{-1}\right) \rightarrow\left(\frac{x_{2}}{\left(1+x_{1}\right)^{2}}, x_{1}^{-1}, 0, \infty\right) .
$$

Again the limiting value of $x_{3}, x_{4}$ is preserved, while in terms of operations of the Kronecker quiver the q-Painlevé flow becomes

$$
T_{\mathbf{F}_{0}}=(1,2) \mu_{1},
$$

which is an automorphism of the Kronecker quiver.

\subsection{Local $\mathbb{F}_{1}$}

We can proceed and take the analogous limit for local $\mathbb{F}_{1}$, for which

$$
t=x_{1} x_{2}^{-1} x_{3}^{2}, \quad q=x_{1} x_{2} x_{3} x_{4} .
$$

We again focus on the Kronecker subquiver with nodes 1,2 , and set

$$
x_{3}=\left(t x_{1}^{-1} x_{2}\right)^{1 / 2} \rightarrow 0, \quad x_{4}=q t^{-1 / 2} x_{1}^{-1 / 2} x_{2}^{-3 / 2} \rightarrow \infty .
$$

The limiting behavior of the mutations is now

$$
\begin{aligned}
& \mu_{1}(\mathbf{x})=\left(x_{1}^{-1}, \frac{x_{2}}{\left(1+x_{1}^{-1}\right)^{2}}, \frac{x_{3}}{1+x_{1}^{-1}},\left(1+x_{1}\right)^{3} x_{4}\right) \rightarrow\left(x_{1}^{-1}, \frac{x_{2}}{\left(1+x_{1}^{-1}\right)^{2}}, 0, \infty\right), \\
& \mu_{2}(\mathbf{x})=\left(x_{1}\left(1+x_{2}\right)^{2}, x_{2}^{-1}, \frac{x_{3}}{1+x_{2}^{-1}}, \frac{x_{4}}{1+x_{2}^{-1}}\right) \rightarrow\left(x_{1}\left(1+x_{2}\right)^{2}, x_{2}^{-1}, 0, \infty\right),
\end{aligned}
$$

which again yields the correct limiting behavior. The q-Painlevé flow does not have a good limiting behavior: however, its square does, since

$$
T_{\mathbb{F}_{1}}^{2}=T_{\mathbb{F}_{0}} \rightarrow(1,2) \mu_{1}
$$



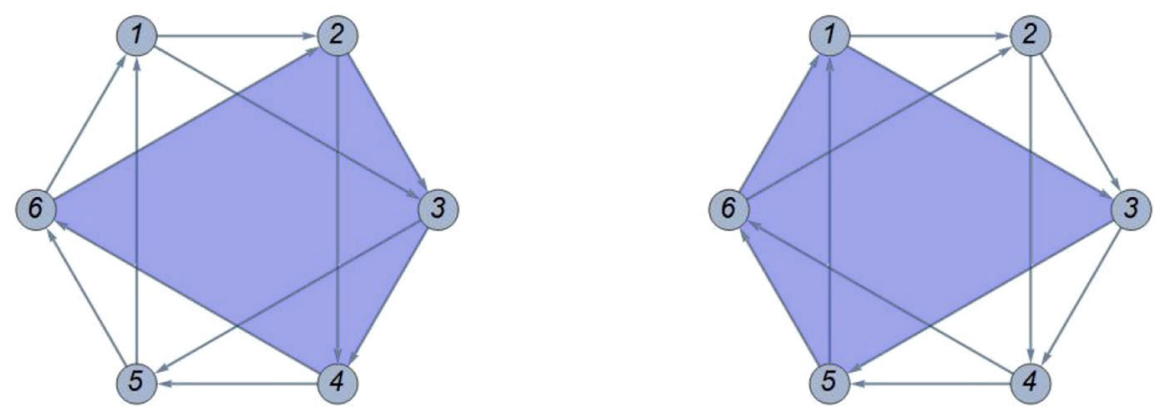

FiguRE 11. $N_{f}=2$ kite-subquivers for the discrete flow $T_{2}$

as we saw above.

\subsection{Local $\mathrm{dP}_{3}$}

This case is much more interesting, because we get different decoupling limits, and only one of them is similar to the usual four-dimensional limit, involving $t \rightarrow 0$. These are related to the presence of different discrete flows. We consider as usual $T_{2}$ first, which we have already seen to be related to the usual weakly coupled/instanton counting picture. In analogy to what was done in the previous cases, since

$$
T_{2}(t)=q t
$$

we take the limit

$$
t \rightarrow 0, \quad q \rightarrow 1,
$$

by taking a limit on two of the cluster variables. Looking at the quiver for this case, we recognize that the subquiver with vertices 2,3,4,6 (or equivalently $1,3,5,6)$ gives the BPS quiver of the four-dimensional $N_{f}=2$ theory, as in Fig. 11. We will focus on the former case.

Because we are "popping out" the nodes 1,5 from the quiver, we want to achieve this by implementing the limit (5.19) directly on the cluster variables. By studying the expressions for the Casimirs (3.32), (3.33), we find

$$
x_{3} x_{6}=Q_{1} Q_{2} q^{1 / 2}, \quad x_{1} x_{4}=\left(Q_{1} Q_{2} t\right)^{-1} \rightarrow \infty, \quad x_{2} x_{5}=q^{1 / 2} t \rightarrow 0,
$$

so that we want to study the limit

$$
x_{1}=\left(x_{4} Q_{1} Q_{2} t\right)^{-1} \rightarrow \infty, \quad x_{5}=q^{1 / 2} t x_{2}^{-1} \rightarrow 0 .
$$

Taking the limit on the mutations, we obtain

$$
\begin{aligned}
\mu_{2}(\mathbf{x}) & =\left(x_{1}\left(1+x_{2}\right), x_{2}^{-1}, \frac{x_{3}}{1+x_{2}^{-1}}, \frac{x_{4}}{1+x_{2}^{-1}}, x_{5},\left(1+x_{2}\right) x_{6}\right) \\
& \rightarrow\left(\infty, x_{2}^{-1}, \frac{x_{3}}{1+x_{2}^{-1}}, \frac{x_{4}}{1+x_{2}^{-1}}, 0,\left(1+x_{2}\right) x_{6}\right)
\end{aligned}
$$

and similarly for the other mutations $\mu_{3}, \mu_{4}, \mu_{6}$ that all degenerate to the mutations of the four-dimensional quiver. The discrete flow also has a "good" 

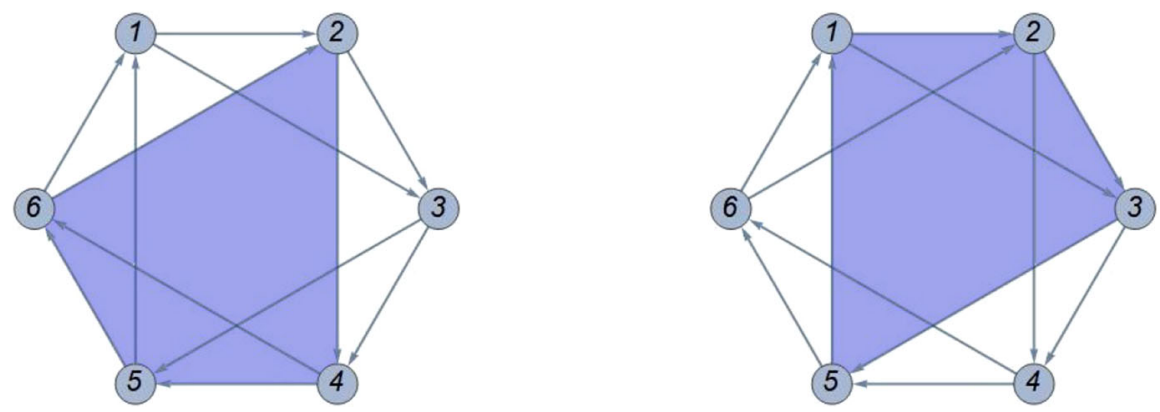

FiguRE 12. $N_{f}=2$ kite-subquivers for the discrete flow $T_{1}$

limit:

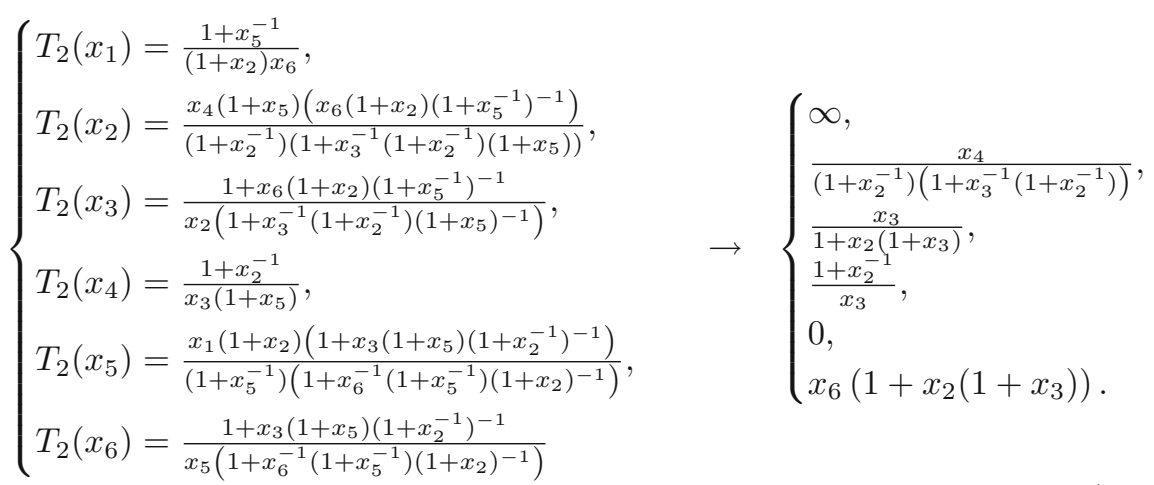

If we call the variables after taking the limit

$$
x_{4} \rightarrow X_{1}, \quad x_{2} \rightarrow X_{2}, \quad x_{3} \rightarrow X_{3}, \quad x_{6} \rightarrow X_{4},
$$

we have that the limit of the discrete flow is

$$
T_{2}^{(4 d)}=(3,2,1) \mu_{3} \mu_{2}
$$

which is an automorphism of the $4 \mathrm{~d}$ quiver. We can follow the same logic for the other discrete flows $T_{1}, T_{3}, T_{4}$ : we will from now on discuss only the limits on the discrete time flows, because those on the mutations are rather simple and given by essentially the same computations as above. In the first case the flow is

$$
T_{1}\left(Q_{+}\right)=q^{-1} Q_{+}, \quad Q_{+}=Q_{1} Q_{2}
$$

so that the natural guess for the right limit to consider is $Q_{+} \rightarrow \infty$, in analogy with the previous case. By looking at the Casimirs, we arrive to the conclusion that we can either decouple the nodes 1,3 or 4,6 , producing the $4 \mathrm{~d} N_{f}=2$ subquivers in Fig. 12.

The limit we want to implement is then

$$
x_{4}=\left(x_{1} Q_{+} t\right)^{-1} \rightarrow 0, \quad x_{6}=Q_{+} q^{1 / 2} x_{3}^{-1} \rightarrow \infty,
$$



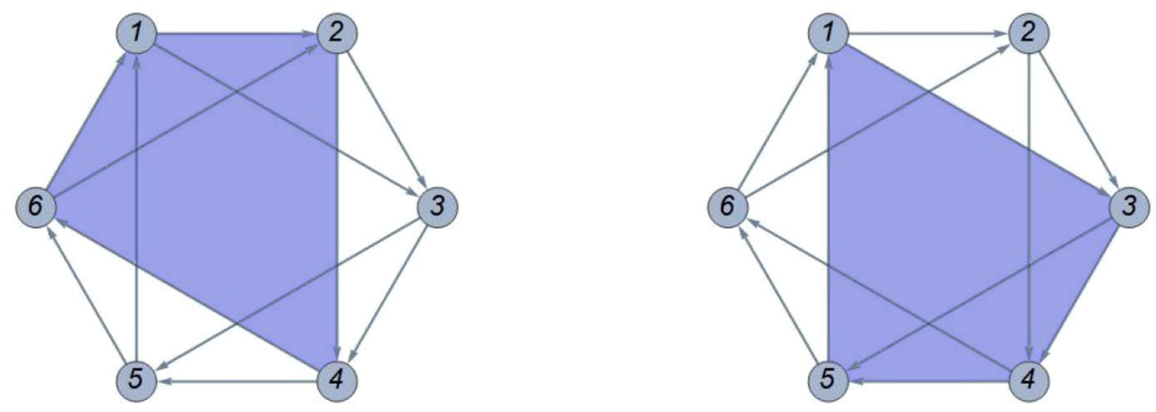

FiguRE 13. $N_{f}=2$ kite-subquivers for the discrete flow $T_{3}$

which gives

$$
\left\{\begin{array} { l } 
{ T _ { 1 } ( x _ { 1 } ) = \frac { x _ { 3 } ( 1 + x _ { 4 } ) ( 1 + x _ { 5 } ( 1 + x _ { 1 } ) ( 1 + x _ { 4 } ^ { - 1 } ) ^ { - 1 } ) } { x _ { 1 } ( 1 + x _ { 2 } ^ { - 1 } ( 1 + x _ { 1 } ^ { - 1 } ) ( 1 + x _ { 4 } ) ^ { - 1 } ) } , } \\
{ T _ { 1 } ( x _ { 2 } ) = \frac { 1 + x _ { 5 } ( 1 + x _ { 1 } ) ( 1 + x _ { 4 } ^ { - 1 } ) ^ { - 1 } } { x _ { 1 } ( 1 + x _ { 2 } ^ { - 1 } ( 1 + x _ { 1 } ^ { - 1 } ) ( 1 + x _ { 4 } ) ^ { - 1 } ) } , } \\
{ T _ { 1 } ( x _ { 3 } ) = \frac { 1 + x _ { 1 } ^ { - 1 } } { x _ { 2 } ( 1 + x _ { 4 } ) } , } \\
{ T _ { 1 } ( x _ { 4 } ) = \frac { x _ { 6 } ( 1 + x _ { 1 } ) ( 1 + x _ { 2 } ( 1 + x _ { 4 } ) ( 1 + x _ { 1 } ^ { - 1 } ) ^ { - 1 } ) } { ( 1 + x _ { 4 } ^ { - 1 } ) ( 1 + x _ { 5 } ^ { - 1 } ( 1 + x _ { 4 } ^ { - 1 } ) ( 1 + x _ { 1 } ) ^ { - 1 } ) } , } \\
{ T _ { 1 } ( x _ { 5 } ) = \frac { 1 + x _ { 2 } ( 1 + x _ { 4 } ) ( 1 + x _ { 1 } ^ { - 1 } ) ^ { - 1 } } { x _ { 4 } ( 1 + x _ { 5 } ^ { - 1 } ( 1 + x _ { 4 } ^ { - 1 } ) ( 1 + x _ { 1 } ) ^ { - 1 } ) } , } \\
{ T _ { 1 } ( x _ { 6 } ) = \frac { 1 + x _ { 4 } ^ { - 1 } } { x _ { 5 } ( 1 + x _ { 1 } ) } }
\end{array} \quad \rightarrow \quad \left\{\begin{array}{l}
\frac{x_{3}}{\left(1+x_{1}^{-1}\right)\left(1+x_{2}^{-1}\left(1+x_{1}^{-1}\right)\right)}, \\
\frac{x_{2}}{1+x_{1}\left(1+x_{2}\right)}, \\
x_{2}^{-1}, \\
x_{5}\left(1+x_{1}\left(1+x_{2}\right)\right), \\
\infty .
\end{array}\right.\right.
$$

which is the same $4 \mathrm{~d}$ quiver automorphism as for $T_{2}$, up to permutations of the nodes. The time evolution $T_{3}$ is characterized by

$$
T_{3}\left(Q_{+}\right)=q Q_{+}, \quad T_{3}(t)=q^{-1} t,
$$

so that the natural limit on the Casimirs is

$$
x_{3} x_{6}=Q_{+} q^{1 / 2} \rightarrow 0, \quad x_{2} x_{5}=q^{1 / 2} t \rightarrow \infty .
$$

This can be achieved by decoupling the nodes 3,5 or 2,6 , keeping the subquivers depicted in Fig. 13.

Choosing the former one for concreteness, we want to compute the limit

$$
x_{3}=Q_{+} q^{1 / 2} x_{6}^{-1} \rightarrow 0, \quad x_{5}=q^{1 / 2} t x_{2}^{-1} \rightarrow \infty
$$


on the discrete evolution $T_{3}$. This is given by

$$
\left\{\begin{array} { l } 
{ T _ { 3 } ( x _ { 1 } ) = \frac { 1 + x _ { 4 } ( 1 + x _ { 6 } ) ( 1 + x _ { 3 } ^ { - 1 } ) ^ { - 1 } } { x _ { 6 } ( 1 + x _ { 1 } ^ { - 1 } ( 1 + x _ { 6 } ^ { - 1 } ) ( 1 + x _ { 3 } ) ) } , } \\
{ T _ { 3 } ( x _ { 2 } ) = \frac { 1 + x _ { 6 } ^ { - 1 } } { x _ { 1 } ( 1 + x _ { 3 } ) } , } \\
{ T _ { 3 } ( x _ { 3 } ) = \frac { x _ { 5 } ( 1 + x _ { 6 } ) ( 1 + x _ { 1 } ( 1 + x _ { 3 } ) ( 1 + x _ { 6 } ^ { - 1 } ) ^ { - 1 } ) } { ( 1 + x _ { 3 } ^ { - 1 } ) ( 1 + x _ { 4 } ( 1 + x _ { 3 } ^ { - 1 } ) ( 1 + x _ { 6 } ) ^ { - 1 } ) } , } \\
{ T _ { 3 } ( x _ { 4 } ) = \frac { 1 + x _ { 1 } ( 1 + x _ { 3 } ) ( 1 + x _ { 6 } ^ { - 1 } ) ^ { - 1 } } { x _ { 3 } ( 1 + x _ { 4 } ^ { - 1 } ( 1 + x _ { 3 } ^ { - 1 } ) ( 1 + x _ { 6 } ) ^ { - 1 } ) } , } \\
{ T _ { 3 } ( x _ { 5 } ) = \frac { 1 + x _ { 3 } ^ { - 1 } } { x _ { 4 } ( 1 + x _ { 6 } ) } , } \\
{ T _ { 3 } ( x _ { 6 } ) = \frac { x _ { 2 } ( 1 + x _ { 3 } ) ( 1 + x _ { 4 } ( 1 + x _ { 6 } ) ( 1 + x _ { 3 } ^ { - 1 } ) ^ { - 1 } ) } { ( 1 + x _ { 6 } ^ { - 1 } ) ( 1 + x _ { 1 } ^ { - 1 } ( 1 + x _ { 6 } ^ { - 1 } ) ( 1 + x _ { 3 } ) ^ { - 1 } ) } }
\end{array} \quad \rightarrow \quad \left\{\begin{array}{l}
\frac{x_{1}}{1+x_{6}\left(1+x_{1}\right)} \\
\frac{1+x_{6}^{-1}}{x_{1}}, \\
0, \\
x_{4}\left(1+x_{6}\left(1+x_{1}\right)\right), \\
\infty, \\
\frac{x_{2}}{\left(1+x_{6}^{-1}\right)\left(1+x_{1}^{-1}\left(1+x_{6}^{-1}\right)\right)}
\end{array}\right.\right.
$$

We see that in all the cases that yielded the time evolution of q-Painlevé $\mathrm{III}_{1}$, the degeneration of the time flow produces the same automorphism of an appropriate subquiver. It remains to study the flow $T_{4}$, which yielded a q-Painlevé IV time evolution, characterized by

$$
T_{4}\left(Q_{-}\right)=q Q_{-}, \quad Q_{-}=\frac{Q_{2}}{Q_{1}} .
$$

In terms of the Casimirs $b_{0}, b_{1}$, this leads to

$$
x_{2} x_{4} x_{6}=\left(q Q_{-}\right)^{1 / 2} \rightarrow 0, \quad x_{1} x_{3} x_{5}=q^{-1} Q_{-}^{-1 / 2} \rightarrow \infty .
$$

To achieve this without affecting the Casimirs $a_{0}, a_{1}, a_{2}$ we have to decouple either the nodes 2,5 , or the nodes 3,6 , or the nodes 1,4 , giving the subquivers in Fig. 14, and we will consider the first option, given by the limit

$$
x_{2}=\left(q Q_{-}\right)^{1 / 2} x_{4}^{-1} x_{6}^{-1} \rightarrow 0, \quad x_{5}=q^{-1} Q_{-}^{-1 / 2} x_{1}^{-1} x_{3}^{-1} \rightarrow \infty .
$$

Here, something similar to what happened when we studied the degeneration of the q-Painlevé $\mathrm{III}_{3}$ associated to local $\mathbb{F}_{1}$ happens: recall that in that case $T_{\mathbb{F}_{1}}$ did not have a good degeneration limit as an automorphism of the subquiver, but rather its square did. We observed that this was related to the $\mathbb{Z}_{2}$-periodicity of the action of $T_{\mathbb{F}_{1}}$ on the BPS charges. What happens here is that not $T_{4}$, but rather $T_{4}^{3}$ has a good action after taking the limit, in particular only for $T_{4}^{3}$ it is true that

$$
T_{4}^{3}\left(x_{2}\right) \rightarrow 0, \quad T_{4}^{3}\left(x_{5}\right) \rightarrow \infty,
$$

consistently with the limit.

The resulting subquiver is the oriented square with arrows of valency one and no diagonals with adjacency matrix

$$
B=\left(\begin{array}{cccc}
0 & 1 & 0 & -1 \\
-1 & 0 & 1 & 0 \\
0 & -1 & 0 & 1 \\
1 & 0 & -1 & 0
\end{array}\right)
$$



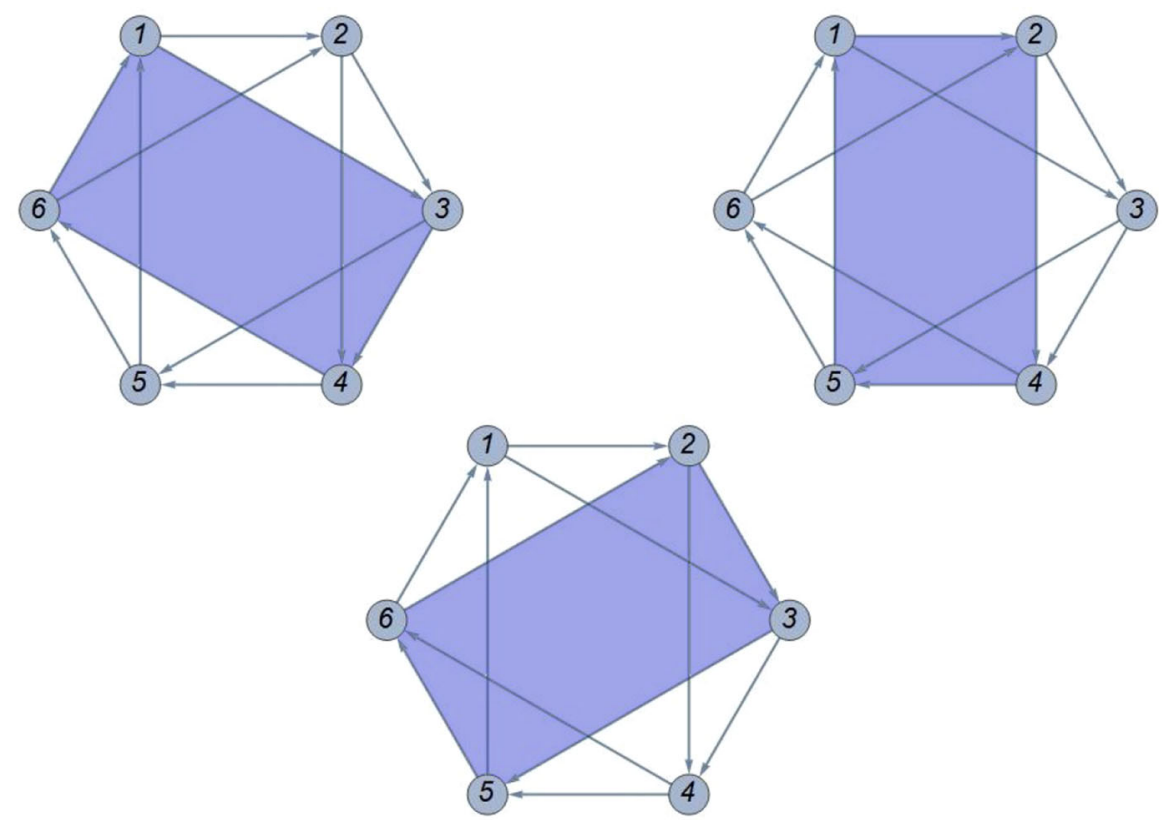

FiguRE 14. $N_{f}=2$ subquivers for the discrete flow $T_{4}$

The corresponding four-dimensional gauge theory has been already classified in [14] as $Q(1,1)$ and shown to correspond to $H_{3}$, which is the ArgyresDouglas limit of the $N_{f}=3$ with $S U(2)$. All this is consistent with the reductions of the Sakai's table in Fig. 1.

The symmetry type of the five-dimensional $S U(2) N_{f}=2$ gauge theory is $E_{3}^{(1)}$. The reduction of the $T_{1}, T_{2}$ and $T_{3}$ flows corresponds to the reduction $E_{3}^{(1)} \rightarrow D_{2}^{(1)}$, the latter being the symmetry type of the four-dimensional $S U(2) N_{f}=2$ gauge theory. The reduction of the $T_{4}$ flow corresponds to the reduction $E_{3}^{(1)} \rightarrow A_{2}^{(1)}$, the latter being the symmetry type of the $H_{3}$ theory. According to Sakai's classification (see Fig. 1) and the analysis in [42], this flow correctly points to the Argyres-Douglas theory of $N_{f}=3$ which is, in the four-dimensional limit, governed by the differential PIV equation.

\section{Conclusions and Outlook}

In this paper, we studied the discrete flows induced by automorphisms of BPS quivers associated to Calabi-Yau geometries engineering five-dimensional quantum field theories. We showed that these flows provide a simple and effective way to determine the BPS spectrum of these theories, producing at the same time a set of bilinear q-difference equations satisfied by the grand canonical partition function of topological string amplitudes. In the rank one case these are known as q-Painlevé equations and admit in a suitable region of 
the moduli space solutions in terms of Nekrasov-Okounkov, or free fermions, partition functions.

A very attractive feature of this approach is that a simple symmetry principle - the symmetry of the BPS quiver - provides strong constraints on the BPS spectrum and contains a rich and deep set of information which goes well beyond the perturbative approaches to the same theories. Indeed, one can show that the non-perturbative completion of topological string via a spectral determinant presentation, arising in the context of the topological string/ spectral theory correspondence, arises naturally as solution of this system of discrete flow equations [9]. Moreover, some of the flows associated to the BPS quiver directly link to non-perturbative phases of the corresponding gauge theory, as we have seen for a particular flow of the local $d P_{3}$ geometry which describe a $\left(A_{1}, D_{4}\right)$ Argyres-Douglas point, see Sect. 5.3. A discussion of the relation between Painlevé equations and Argyres-Douglas points of fourdimensional gauge theories can be found in [42] based on the class $\mathcal{S}$ description of these theories [49].

It is also very interesting that a fully classical construction, the cluster algebra associated to the BPS quiver, contains information about the quantum geometry of the Calabi-Yau. Indeed, the zeroes of the $\tau$-functions of the cluster algebra provide the exact spectrum of the associated quantum integrable system, as it was shown in [9] for the local $\mathbb{F}_{0}$ geometry corresponding to relativistic Toda chain [50], and in [51,52] for its four-dimensional/non-relativistic limit. Further evidence we provide in this paper is that the $\mathrm{X}$-cluster variables of the $5 \mathrm{~d}$ quiver flow in the $4 \mathrm{~d}$ limit to the X-cluster variables of the corresponding four-dimensional BPS quiver, which are known to be related to the Voros symbols, i.e. exponential of the exact quantum WKB periods of the fourdimensional integrable system [53]. The fact the we find that standard topological string - or equivalently 5 d gauge theory in the self-dual $\Omega$-background $\epsilon_{1}+\epsilon_{2}=0$ rather than in the Nekrasov-Shatashvili [54] background $\epsilon_{1}=0$ provides a quantization of the Calabi-Yau geometry is not fully surprising from the view point of equivariant localization. Indeed, the difference between the two cases resides in a different choice of one-parameter subgroup of the full toric action, and therefore contains the same amount of information, although under possibly very non-trivial combinatorial identities. A first instance of this phenomenon was discussed from the mathematical perspective in [55]. For the case at hand, the non-trivial relation between the two approaches is encoded in a suitable limit of blow-up equations [43,56-59].

There are several directions to further investigate.

Let us notice that, with respect to the framework of $[13,31]$, to define a BPS chamber one should set the precise order of the arguments of the central charges $Z\left(\gamma_{i}\right)$ for all the charges $\gamma_{i}$ in the spectrum. While our method efficiently computes the spectrum, at least in the tame chambers, it doesn't point yet to a precise definition of the corresponding moduli values. This is because we still miss a link with the relevant stability conditions. Our method relies on the existence of patches in the moduli space where the topological 
string partition function allows finite radius converging expansions ${ }^{8}$. Let us notice that clarifying this point would prepare the skeleton of the demonstration that Kontsevich-Soibelman wall-crossing would be equivalent to the discrete equations (q-Painlevé and higher rank analogues) we obtain. Moreover, the chambers we do compute are "triangular" in the sense of [60]. In this paper, it is shown that similar chambers exist for all the class $\mathcal{S}\left[A_{1}\right]$ theories: while multiple affinizations are generically involved, these coincide with the ones computed with our methods, at least in the examples we work out explicitly.

We have seen that when the Calabi-Yau geometry admits several moduli there are various inequivalent flows for the same BPS quiver, but only few of them have a realization in terms of weakly coupled Lagrangian field theories. In some cases the other flows correspond simply to Bäcklund transformations mapping solutions one into the others. This is the case, for example, for the fluxes $T_{1}, T_{2}, T_{3}$ discussed in Sect. 3.3. We expect that the full solution to this system of equations will be given in terms of suitable expansions of the topological vertex ${ }^{9}[3]$, while its non-perturbative completion should be given by the spectral determinant of the corresponding $N_{f}=2$ spectral curve. In other cases the flows are intrinsically non-perturbative, like the flux $T_{4}$ discussed in Sect. 5.3. It would be interesting to characterize the solutions of these flows in terms of supersymmetric indices of four-dimensional gauge theories [63-65].

We expect that the full refined topological string or equivalently the gauge theory in the full $\Omega$ background is captured by the quantum cluster algebra. The bilinear equations in this case are expected to have a direct relation to the K-theoretic blow-up equations [24].

We have also shown that the X-cluster variables correctly reproduce the ones of the four-dimensional BPS quivers under a suitable scaling limit. It would be very interesting to further explore the relation of our results with the ones on exact WKB methods and TBA equations [56,66,67], possibly extending these methods to the q-difference/5d case. For the class $\mathrm{S}$ theories, an important rôle should be played by the group Hitchin system [68], in the perspective of its quantization $[69,70]$. An interesting open question is the nature of the quantum periods in five dimensions and their relation to the cluster variables appearing in the study of q-Painlevé equations.

In the four-dimensional case, the Painlevé/gauge theory correspondence [42] extends also to non-toric cases, corresponding to isomonodromic deformation problems on higher genus Riemann surfaces, see [71,72] for the genus one case. These have a $5 \mathrm{~d}$ uplift in terms of q-Virasoro algebra [73] and matrix models $[74,75]$ whose BPS quiver interpretation would be more than welcome. Also the higher rank extension of BPS quiver flows and the associated tau-functions is to be explored in detail. As a first example, one can consider $S U(N) \mathrm{Su}-$ per Yang-Mills, whose spectral determinant in matrix model presentation was presented in [76]. In the one period phase, this satisfies $N$-particle Toda chain equations. The corresponding cluster integrable system is discussed in [19].

\footnotetext{
${ }^{8}$ See also the comments at the end of the Sect. 4.2 on this point.

${ }^{9}$ Similar considerations appeared in $[61,62]$ for the four-dimensional case.
} 
More in general, our method should extend beyond the rank 1 case and qPainlevé systems, pointing to more general results about topological string partition functions and discrete dynamical systems.

\section{Acknowledgements}

We would like to thank M. Cirafici, D. Fioravanti, M. Semenyakin, and A. Marshakov for interesting discussions. Many thanks also to M. Del Zotto and P. Gavrylenko for a careful reading of the manuscript and interesting comments. The research of G.B. and F.DM. is partly supported by the INFN Iniziativa Specifica ST\&FI and by the PRIN project "Non-perturbative Aspects Of Gauge Theories And Strings". The research of A.T. is partly supported by the INFN Iniziativa Specifica GAST and by the PRIN project "Geometria delle varietà algebriche".

Funding Open access funding provided by Scuola Internazionale Superiore di Studi Avanzati - SISSA within the CRUI-CARE Agreement.

Open Access. This article is licensed under a Creative Commons Attribution 4.0 International License, which permits use, sharing, adaptation, distribution and reproduction in any medium or format, as long as you give appropriate credit to the original author(s) and the source, provide a link to the Creative Commons licence, and indicate if changes were made. The images or other third party material in this article are included in the article's Creative Commons licence, unless indicated otherwise in a credit line to the material. If material is not included in the article's Creative Commons licence and your intended use is not permitted by statutory regulation or exceeds the permitted use, you will need to obtain permission directly from the copyright holder. To view a copy of this licence, visit http://creativecommons. org/licenses/by/4.0/.

Publisher's Note Springer Nature remains neutral with regard to jurisdictional claims in published maps and institutional affiliations.

\section{A. q-Special Functions and Nekrasov Functions}

The $5 \mathrm{~d}$ instanton partition function $[77,78]$ for $\mathcal{N}=15 \mathrm{~d} S U(N)$ SYM with $N_{f}$ fundamental flavors and Chern-Simons level $k=0,1, \ldots, N-1$ is given by a sum over $\mathrm{N}$-tuples if partitions $\boldsymbol{\lambda}\left(\lambda_{1}, \ldots, \lambda_{N}\right)$ with counting parameter $t$ :

$$
Z_{\text {inst }}=\sum_{\lambda} t^{|\lambda|} Z_{\lambda}^{C S} Z_{\lambda}^{\text {fund }} Z_{\lambda}^{\text {gauge }} .
$$

The Chern-Simons factor is given by

$$
Z_{\lambda}^{C S}=\prod_{i=1}^{N} T_{\lambda_{i}}\left(u_{i} ; q_{1}, q_{2}\right)^{k}, \quad T_{\lambda}\left(u ; q_{1}, q_{2}\right)=\prod_{(i, j) \in \lambda} u q_{1}^{i-1} q_{2}^{j-1},
$$


while the matter and gauge contributions can all be written in terms of the single building block

$$
N_{\lambda, \mu}\left(u, q_{1}, q_{2}\right)=\prod_{s \in \lambda}\left(1-u q_{2}^{-a_{\mu}(s)-1} q_{1}^{l_{\lambda}(s)}\right) \prod_{s \in \mu}\left(1-u q_{2}^{a_{\lambda}(s)} q_{1}^{-l_{\mu}(s)-1}\right),
$$

in the following way:

$$
\begin{aligned}
Z_{\lambda}^{\text {fund }} & =\prod_{i=1}^{N_{f}} \prod_{\alpha=1}^{N} N_{\lambda, \emptyset}\left(Q_{i} u_{\alpha}\right), \\
Z_{\lambda}^{\text {gauge }} & =\prod_{i, j=1}^{N} \frac{1}{N_{\lambda_{i}, \lambda_{j}}\left(u_{i} / u_{j} ; q_{1}, q_{2}\right)} .
\end{aligned}
$$

The perturbative contribution is given by the following:

$$
\begin{aligned}
Z_{c l} & =e^{-\log t \frac{\sum_{i=1}^{N}\left(\log u_{i}\right)^{2}}{2 \log q_{1} \log q_{2}}-k \frac{\sum_{i=1}^{N}\left(\log u_{i}\right)^{3}}{6 \log q_{1} \log q_{2}}}, \\
Z_{1 \text {-loop }} & =\frac{\prod_{1 \leq \alpha \neq \beta \leq N}\left(u_{\alpha} / u_{\beta} ; q_{1}, q_{2}\right)_{\infty}}{\prod_{i=1}^{N_{f}} \prod_{\alpha=1}^{N}\left(Q_{i} u_{\alpha} ; q_{1}, q_{2}\right)_{\infty}} .
\end{aligned}
$$

Here $\left(u_{i} / u_{j} ; q_{1}, q_{2}\right)_{\infty}$ is the multiple q-Pochhammer symbol, defined by

$$
\left(z ; q_{1}, \ldots, q_{n}\right)_{\infty} \equiv \prod_{i_{1}, \ldots, i_{N}=0}^{\infty}\left(1-z \prod_{k=1}^{n} q_{k}^{i_{k}}\right)=\exp \left(-\sum_{m=1}^{\infty} \frac{z^{m}}{m} \prod_{k=1}^{N} \frac{1}{1-q_{k}^{m}}\right)
$$

In all the formulae above, the following notations are used, in terms of the four-dimensional gauge theory parameters:

$$
u_{\alpha}=e^{\beta a_{\alpha}}, \quad Q_{i}=e^{-\beta m_{i}}, \quad q_{1}=e^{\beta \epsilon_{1}}, \quad q_{2}=e^{\beta \epsilon_{2}} .
$$

An important property of the double Pochhammer symbol that has to be used repeatedly when solving the bilinear equations, is the following:

$$
\begin{array}{ll}
\frac{\left(z q ; q, q^{-1}\right)_{\infty}}{\left(z ; q, q^{-1}\right)_{\infty}}=(z q ; q)_{\infty}, & \frac{\left(z q^{-1} ; q, q^{-1}\right)_{\infty}}{\left(z ; q, q^{-1}\right)_{\infty}}=\frac{1}{(z ; q)_{\infty}}, \\
\frac{(z q ; q,)_{\infty}}{(z ; q)_{\infty}}=\frac{1}{1-z} &
\end{array}
$$

The full partition function $Z(u ; t)$ is given by $Z=Z_{c l} Z_{1-\text { loop }} Z_{\text {inst }}$.

\section{B. q-Painlevé III and IV in Tsuda's Parameterization}

We provide here the choice of parameters for the cluster algebra that reproduces the q-Painlevè $I I I_{1}$ and $I V$ equations of [30]. It turns out for this to be useful to introduce

$$
u_{1}=a_{1}\left(\frac{b_{1}}{b_{0}}\right)^{1 / 3}, \quad u_{2}=a_{2}\left(\frac{b_{0}}{b_{1}}\right)^{1 / 3}, \quad u_{3}=a_{0}\left(\frac{b_{1}}{b_{0}}\right)^{1 / 3}
$$




$$
u_{4}=a_{1}\left(\frac{b_{0}}{b_{1}}\right)^{1 / 3}, \quad u_{5}=a_{2}\left(\frac{b_{1}}{b_{0}}\right)^{1 / 3}, \quad u_{6}=a_{0}\left(\frac{b_{0}}{b_{1}}\right)^{1 / 3}
$$

Note that in [30] the Casimirs have a geometric meaning in terms of points blown-up on $\mathbb{P}^{1} \times \mathbb{P}^{1}$.

\section{B.1. qPIII}

Let us consider first the case of qPIII. We choose as basis for the tropical semifield four independent Casimirs and parameterize in terms of them the $y_{i}$ 's in such a way that (3.32) and (3.33) are satisfied, together with the correct time evolution (3.37). We choose as independent Casimirs $a_{1}, a_{2}, q, b_{0}$. To match with [30], we also have to make a different choice for the time evolution parameter $q$,

$$
q=\prod_{i} y_{i}^{-1 / 2}
$$

Set

$$
\begin{aligned}
& y_{1}=q^{-1 / 3} b_{0}^{2 / 3} a_{1}^{-1}, \quad y_{2}=q^{1 / 3} b_{0}^{2 / 3} a_{2}^{-1}, \quad y_{3}=q^{-4 / 3} b_{0}^{2 / 3} a_{1} a_{2}, \\
& y_{4}=q^{1 / 3} b_{0}^{-2 / 3} a_{1}^{-1}, \quad y_{5}=q^{-1 / 3} b_{0}^{2 / 3} a_{2}^{-1}, \quad y_{6}=q^{-2 / 3} b_{0}^{-2 / 3} a_{1} a_{2} .
\end{aligned}
$$

The time evolution is the following (we only write the relevant $\tau$-variables):

$$
\left\{\begin{array}{l}
\overline{\tau_{2}}=\tau_{4}, \\
\overline{\tau_{3}}=\frac{a_{2} b_{0}^{2 / 3} \tau_{3} \tau_{4}+q^{1 / 3} \tau_{1} \tau_{6}}{\tau_{2}}, \\
\overline{\tau_{5}}=\tau_{1}, \\
\overline{\tau_{6}}=\frac{q^{1 / 3} a_{2} \tau_{1} \tau_{6}+\tau_{3} \tau_{4} b_{0}^{2 / 3}}{\tau_{5}},
\end{array}, \quad\left\{\begin{array}{l}
\underline{\tau_{1}}=\tau_{5}, \\
\underline{\tau_{3}}=\frac{a_{1} b_{0}^{2 / 3} \tau_{5} \tau_{6}+q^{1 / 3} \tau_{2} \tau_{3}}{\tau_{4}}, \\
\underline{\tau_{4}}=\tau_{2}, \\
\underline{\tau_{6}}=\frac{q^{1 / 3} a_{1} \tau_{2} \tau_{3}+b_{0}^{2 / 3} \tau_{5} \tau_{6}}{,}
\end{array}\right.\right.
$$

leading to the bilinear equations

$$
\begin{array}{lll}
\overline{\tau_{3}} \tau_{2}=q^{1 / 3}\left(\overline{\tau_{5}} \tau_{6}+u_{2} \tau_{3} \overline{\tau_{2}}\right), & \overline{\tau_{6}} \tau_{5}=b_{0}^{2 / 3}\left(u_{5} \overline{\tau_{5}} \tau_{6}+\tau_{3} \overline{\tau_{2}}\right), \\
\overline{\tau_{2}} \underline{\tau_{3}}=q^{1 / 3}\left(u_{4} \tau_{5} \tau_{6}+\tau_{2} \tau_{3}\right), & \overline{\tau_{5}} \underline{\tau_{6}}=b_{0}^{2 / 3}\left(u_{1} \tau_{2} \tau_{3}+\tau_{5} \tau_{6}\right) .
\end{array}
$$

These differ from the bilinear equations of [30] by different overall factors of the RHS, so in principle it would seem that they are different bilinear equations. However, they are still equivalent to qPIII. If we define

$$
f=\frac{\tau_{5} \tau_{6}}{\tau_{2} \tau_{3}}, \quad g=\frac{\tau_{1} \tau_{6}}{\tau_{3} \tau_{4}},
$$

we get the system of first-order q-difference equations

$$
\bar{f} f=a_{2} g \frac{g+u_{5}^{-1}}{g+u_{2}}, \quad \bar{g} g=\frac{f}{a_{1}} \frac{f+u_{1}}{f+u_{4}^{-1}},
$$

which is the qPIII equation appearing in [30]. 


\section{B.2. qPIV}

The action of $T_{4}$ on the tau function is the following:

$$
\begin{aligned}
& \left\{\begin{array}{l}
\overline{\tau_{1}}=\tau_{4}, \\
\overline{\tau_{2}}=\frac{q^{4 / 3} b_{0}^{2 / 3} \tau_{4} \tau_{5} \tau_{6}+a_{1} q^{5 / 3} \tau_{2} \tau_{3} \tau_{4}+a_{1} a_{2} b_{0}^{4 / 3} \tau_{1} \tau_{2} \tau_{6}}{\tau_{1} \tau_{3}}, \\
\overline{\tau_{3}}=\tau_{6}, \\
\overline{\tau_{4}}=\frac{q^{5 / 3} \tau_{4} \tau_{5} \tau_{6}+a_{1} b_{0}^{4 / 3} \tau_{2} \tau_{3} \tau_{4}+a_{1} a_{2} q^{1 / 3} b_{0}^{2 / 3} \tau_{1} \tau_{2} \tau_{6}}{\tau_{3} \tau_{5}}, \\
\overline{\tau_{5}}=\tau_{2}, \\
\overline{\tau_{6}}=\frac{b_{0}^{4 / 3} \tau_{4} \tau_{5} \tau_{6}+q^{1 / 3} a_{1} b_{0}^{2 / 3} \tau_{2} \tau_{3} \tau_{4}+q^{2 / 3} a_{1} a_{2} \tau_{1} \tau_{2} \tau_{6}}{\tau_{1} \tau_{5}},
\end{array}\right. \\
& \left\{\begin{array}{l}
\underline{\tau_{1}}=\frac{q^{2 / 3} b_{0}^{4 / 3} \tau_{1} \tau_{2} \tau_{3}+q^{1 / 3} a_{1} \tau_{1} \tau_{5} \tau_{6}+a_{1} a_{2} b_{0}^{2 / 3} \tau_{3} \tau_{4} \tau_{5}}{\tau_{2} \tau_{6}}, \\
\underline{\tau_{2}}=\tau_{5}, \\
\underline{\tau_{3}}=\frac{q^{2 / 3} \tau_{1} \tau_{2} \tau_{3}+q^{1 / 3} a_{1} b_{0}^{2 / 3} \tau_{1} \tau_{5} \tau_{6}+a_{1} a_{2} b_{0}^{4 / 3} \tau_{3} \tau_{4} \tau_{5}}{\tau_{2} \tau_{4}}, \\
\underline{\tau_{4}}=\tau_{1}, \\
\underline{\tau_{5}}=\frac{q^{2 / 3} b_{0}^{2 / 3} \tau_{1} \tau_{2} \tau_{3}+q^{1 / 3} a_{1} b_{0}^{4 / 3} \tau_{1} \tau_{5} \tau_{6}+a_{1} a_{2} \tau_{3} \tau_{4} \tau_{5}}{\tau_{4} \tau_{6}}, \\
\underline{\tau_{6}}=\tau_{3} .
\end{array}\right.
\end{aligned}
$$

At a first glance, these seem trilinear, rather than bilinear, equations. However, take linear combinations of (B.11) in such a way that the first term on the RHS cancels out:

$$
\left\{\begin{array}{l}
\overline{\tau_{6}} \tau_{1} b_{0}^{-4 / 3}-q^{-5 / 3} \overline{\tau_{4}} \tau_{3}=\frac{\tau_{2} \tau_{3} \tau_{4} a_{1}\left(q^{1 / 3} b_{0}^{-2 / 3}-q^{-5 / 3} b_{0}^{4 / 3}\right)+a_{1} a_{2} \tau_{1} \tau_{2} \tau_{6}\left(q^{2 / 3} b_{0}^{-4 / 3}-q^{-4 / 3}\right)}{\tau_{5}}, \\
\overline{\tau_{6}} \tau_{5} b_{0}^{-2 / 3}-q^{-4 / 3} \bar{\tau}_{2} \tau_{3}=\frac{a_{1} a_{2} \tau_{1} \tau_{2} \tau_{6}\left(q^{2 / 3} b_{0}^{-2 / 3}-q^{-4 / 3} b_{0}^{4 / 3}\right)}{\tau_{1}}, \\
\overline{\tau_{4}} \tau_{5} q^{-1 / 3}-b_{0}^{-2 / 3} \overline{\tau_{2}} \tau_{1}=\frac{\tau_{2} \tau_{3} \tau_{4} a_{1} q^{4 / 3}\left(q^{-5 / 3} b_{0}^{4 / 3}-q^{1 / 3} b_{0}^{-2 / 3}\right)+a_{1} a_{2} \tau_{1} \tau_{2} \tau_{6}\left(1-b_{0}^{2 / 3}\right)}{\tau_{3}} .
\end{array}\right.
$$

We see that the second equation is now bilinear! We can repeat this procedure to obtain three bilinear equations from (B.11):

$$
\begin{aligned}
& \bar{\tau}_{6} \tau_{5} b_{0}^{-2 / 3}-\overline{\tau_{2}} \tau_{3} q^{-4 / 3}=a_{1} a_{2}\left(q^{2 / 3} b_{0}^{-2 / 3}-b_{0}^{4 / 3} q^{-4 / 3}\right) \tau_{2} \tau_{6}, \\
& \overline{\tau_{6}} \tau_{1} q^{-1 / 3}-\overline{\tau_{4}} \tau_{3} b_{0}^{-2 / 3}=\left(b_{0}^{4 / 3} q^{-1 / 3}-q^{5 / 3} b_{0}^{-2 / 3}\right) \tau_{4} \tau_{6}, \\
& \overline{\tau_{4}} \tau_{5} q^{-1 / 3}-\overline{\tau_{2}} \tau_{1} b_{0}^{-2 / 3}=a_{1}\left(b_{0}^{4 / 3} q^{-1 / 3}-q^{5 / 3} b_{0}^{-2 / 3}\right) \tau_{2} \tau_{4} .
\end{aligned}
$$

We can make these three second-order equations in three variables by using the (B.12):

$$
\begin{aligned}
& \bar{\tau}_{6} \underline{\tau_{2}} b_{0}^{-2 / 3}-\overline{\tau_{2}} \underline{\tau_{6}} q^{-4 / 3}=a_{1} a_{2}\left(q^{2 / 3} b_{0}^{-2 / 3}-b_{0}^{4 / 3} q^{-4 / 3}\right) \tau_{2} \tau_{6}, \\
& \overline{\tau_{6}} \underline{\tau_{4}} q^{-1 / 3}-\overline{\tau_{4}} \underline{\tau_{6}} b_{0}^{-2 / 3}=\left(b_{0}^{4 / 3} q^{-1 / 3}-q^{5 / 3} b_{0}^{-2 / 3}\right) \tau_{4} \tau_{6}, \\
& \overline{\tau_{4}} \underline{\tau_{2}} q^{-1 / 3}-\overline{\tau_{2}} \underline{\tau_{4}} b_{0}^{-2 / 3}=a_{1}\left(b_{0}^{4 / 3} q^{-1 / 3}-q^{5 / 3} b_{0}^{-2 / 3}\right) \tau_{2} \tau_{4},
\end{aligned}
$$

These are the bilinear equations for qPIV, with the parameterization of $[30]$. 


\section{C. q-Painlevé Transcendents and Autonomous Limit}

Recall from Sect. 4.1 that the solution $G(t ; q, u, s)$ to the q-Painlevé $\mathrm{III}_{3}$ equation

$$
G(q t) G\left(q^{-1} t\right)=\left(\frac{G(t)+t}{G(t)+1}\right)^{2}
$$

was expressed in terms of cluster X-variables as $G=x_{2}^{-1}$. In terms of dual Nekrasov functions, this translates to

$$
G=i t^{1 / 4} \frac{Z_{1 / 2}^{D}}{Z_{0}^{D}}
$$

The dual partition functions here are defined as

$$
Z_{0}^{D}=\sum_{n \in \mathbb{Z}} s^{n} Z\left(u q^{n}, t\right), \quad Z_{1 / 2}^{D}=\sum_{n \in \mathbb{Z}} s^{n+\frac{1}{2}} Z\left(u q^{n+\frac{1}{2}}, t\right),
$$

where $Z$ is the partition function of pure five-dimensional $S U(2)$ super YangMills. It is possible to study the autonomous limit of this object, following the discussion of [19] for the tau functions. One sets

$$
s=e^{\eta / \hbar}, \quad q=e^{\hbar}, \quad u=e^{a},
$$

sending $\hbar \rightarrow 0$. In this limit, one can write the partition function as

$$
Z(a, \hbar, t)=\exp \left\{\frac{1}{\hbar^{2}} \sum_{n=0}^{\infty} \hbar^{2 n} F_{n}(a, t)\right\}
$$

so that we want to take the "semi-classical" (actually autonomous) $\hbar \rightarrow 0$ limit of the dual partition functions, keeping the leading and first subleading term. Let us review the argument: in [7] it was shown that the saddle point of $Z$ is the Seiberg-Witten A-period: let us denote this by $a_{*}$. It is such that

$$
\eta=-F_{0}^{\prime}\left(a_{*}\right)+\mathcal{O}\left(\hbar^{2}\right)
$$

The sum over $n$ will be dominated by terms that are close to this saddle point: let us denote by $n_{*}$ the value of $n$ such that $a+\hbar n$ is closest to $a_{*}$ : in other words, set

$$
a+\hbar n_{*} \equiv a_{*}+\hbar x, \quad x \sim \mathcal{O}(1) .
$$

To expand $Z_{0}^{D}$ around such value of $n$, we set $n=n_{*}+k$ and we sum over $k$. We also invert the relation for $n_{*}$,

$$
n_{*}=\frac{a_{*}-a}{\hbar}+x
$$


in order to parameterize everything in terms of the UV Cartan parameter $a$ and the IR A-period $a_{*}$. The resulting saddle point expansion for $Z_{0}^{D}$ is

$$
\begin{aligned}
Z_{0}^{D}= & \sum_{k} \exp \left\{\frac{1}{\hbar}\left(n_{*}+k\right) \eta+\frac{1}{\hbar^{2}} F_{0}\left(a_{*}+\hbar(k+x)\right)+F_{1}\left(a_{*}+\hbar(k+x)\right)\right\} \\
= & \sum_{k}\left\{\frac{1}{\hbar^{2}}\left[F_{0}\left(a_{*}\right)+\left(a_{*}-a\right) \eta\right]+\frac{1}{\hbar}\left[(x+k) \eta+(x+k) F_{0}^{\prime}\left(a_{*}\right)\right]\right\} \\
& \times \exp \left\{\frac{1}{2}(x+k)^{2} F_{0}^{\prime \prime}\left(a_{*}\right)+F_{1}\left(a_{*}\right)\right\} \\
= & e^{\frac{1}{\hbar^{2}}\left[\left(a_{*}-a\right) \eta+F_{0}\left(a_{*}\right)\right]+F_{1}\left(a_{*}\right)+i \pi \tau_{S W} x^{2}} \vartheta_{3}\left(\tau_{S W} x \mid \tau_{S W}\right) e^{\mathcal{O}(\hbar)},
\end{aligned}
$$

where we defined the IR coupling constant

$$
2 \pi i \tau_{S W}=F_{0}^{\prime \prime}\left(a_{*}\right) \text {. }
$$

An analogous computation can be done for the half-integer Fourier series:

$$
\begin{aligned}
Z_{1 / 2}^{D}= & \sum_{k} \exp \left\{\frac{1}{\hbar}\left(n_{*}+k+1 / 2\right) \eta\right. \\
& \left.+\frac{1}{\hbar^{2}} F_{0}\left(a_{*}+\hbar(k+1 / 2+x)\right)+F_{1}\left(a_{*}+\hbar(k+1 / 2+x)\right)\right\} \\
= & \sum_{k}\left\{\frac{1}{\hbar^{2}}\left[F_{0}\left(a_{*}\right)+\left(a_{*}-a\right) \eta\right]+\frac{1}{\hbar}\left[(x+k+1 / 2) \eta+(x+k+1 / 2) F_{0}^{\prime}\left(a_{*}\right)\right]\right\} \\
& \times \exp \left\{\frac{1}{2}(x+k+1 / 2)^{2} F_{0}^{\prime \prime}\left(a_{*}\right)+F_{1}\left(a_{*}\right)\right\} \\
= & e^{\frac{1}{\hbar^{2}}\left[\left(a_{*}-a\right) \eta+F_{0}\left(a_{*}\right)\right]+F_{1}\left(a_{*}\right)+i \pi \tau_{S W} x^{2}} \vartheta_{2}\left(\tau_{S W} x \mid \tau_{S W}\right) e^{\mathcal{O}(\hbar)} .
\end{aligned}
$$

When we take the ratio of these two expressions, the overall factors simplify, giving

$$
G=x_{2}^{-1}=i t^{1 / 4} \frac{\vartheta_{2}\left(\tau_{S W} x \mid \tau_{S W}\right)}{\vartheta_{3}\left(\tau_{S W} x \mid \tau_{S W}\right)} e^{\mathcal{O}(\hbar)} .
$$

\section{References}

[1] Hori, K.: Mirror Symmetry, Clay Mathematics Monographs. American Mathematical Society (2003)

[2] Iqbal, A., Vafa, C.: BPS degeneracies and superconformal index in diverse dimensions. Phys. Rev. D 90, 105031 (2014). arXiv:1210.3605

[3] Aganagic, M., Klemm, A., Marino, M., Vafa, C.: The topological vertex. Commun. Math. Phys. 254, 425 (2005). arXiv:hep-th/0305132

[4] Dijkgraaf, R., Hollands, L., Sulkowski, P., Vafa, C.: Supersymmetric gauge theories, intersecting branes and free fermions. JHEP 02, 106 (2008). arXiv:0709.4446

[5] Sakai, H.: Rational surfaces associated with affine root systems and geometry of the Painlevé equations. Commun. Math. Phys. 220, 165 (2001)

[6] Seiberg, N.: Five-dimensional SUSY field theories, nontrivial fixed points and string dynamics. Phys. Lett. B 388, 753 (1996). arXiv:hep-th/9608111 
[7] Nekrasov, N., Okounkov, A.: Seiberg-Witten theory and random partitions. Prog. Math. 244, 525 (2006). arXiv:hep-th/0306238

[8] Grassi, A., Hatsuda, Y., Marino, M.: Topological strings from quantum mechanics. Annales Henri Poincare 17, 3177 (2016). arXiv:1410.3382

[9] Bonelli, G., Grassi, A., Tanzini, A.: Quantum curves and $q$-deformed Painlevé equations. Lett. Math. Phys. 109, 1961 (2019). arXiv:1710.11603

[10] Zamolodchikov, A.B.: Painleve III and 2-d polymers. Nucl. Phys. B 432, 427 (1994). arXiv:hep-th/9409108

[11] Tracy, C.A., Widom, H.: Proofs of two conjectures related to the thermodynamic Bethe ansatz. Commun. Math. Phys. 179, 667 (1996). arXiv:solv-int/9509003

[12] Cecotti, S., Vafa, C.: Classification of complete $\mathrm{N}=2$ supersymmetric theories in 4 dimensions. Surveys in differential geometry 18 (2013). arXiv:1103.5832

[13] Alim, M., Cecotti, S., Cordova, C., Espahbodi, S., Rastogi, A., Vafa, C.: BPS quivers and spectra of complete $\mathrm{N}=2$ quantum field theories. Commun. Math. Phys. 323, 1185 (2013). arXiv:1109.4941

[14] Cecotti, S., Del Zotto, M.: $Y$ systems, $Q$ systems, and $4 \mathrm{D} \mathcal{N}=2$ supersymmetric QFT. J. Phys. A 47, 474001 (2014). arXiv:1403.7613

[15] Cirafici, M., Del Zotto, M.: Discrete integrable systems, supersymmetric quantum mechanics, and framed BPS states-I. arXiv:1703.04786

[16] Kontsevich, M., Soibelman, Y.: Stability structures, motivic Donaldson-Thomas invariants and cluster transformations. arXiv:0811.2435

[17] Closset, C., Del Zotto, M.: On 5d SCFTs and their BPS quivers. Part I: B-branes and brane tilings. arXiv:1912.13502

[18] Bershtein, M., Gavrylenko, P., Marshakov, A.: Cluster integrable systems, q-Painlevé equations and their quantization. JHEP 02, 077 (2018). arXiv:1711.02063

[19] Bershtein, M., Gavrylenko, P., Marshakov, A.: Cluster Toda chains and Nekrasov functions, arXiv:1804.10145

[20] Marshakov, A., Semenyakin, M.: Cluster integrable systems and spin chains. JHEP 10, 100 (2019). arXiv:1905.09921

[21] Bershtein, M.A., Shchechkin, A.I.: q-deformed Painlevé $\tau$ function and qdeformed conformal blocks. J. Phys. A 50, 085202 (2017). arXiv:1608.02566

[22] Goncharov, A., Kenyon, R.: Dimers and cluster integrable systems. arXiv: 1107.5588

[23] Yamazaki, M.: Brane Tilings and Their Applications, other thesis (2008). https://doi.org/10.1002/prop.200810536

[24] Bershtein, M., Shchechkin, A.: Painleve equations from Nakajima-Yoshioka blowup relations. arXiv:1811.04050

[25] Jimbo, M., Nagoya, H., Sakai, H.: Cft approach to the q-painlevé vi equation. J. Integrable Syst. 2, 1-27 (2017)

[26] Matsuhira, Y., Nagoya, H.: Combinatorial expressions for the tau functions of $q$-Painlevé V and III equations. arXiv:1811.03285

[27] Nagoya, H.: On $q$-isomonodromic deformations and $q$-Nekrasov functions. arXiv:2004.13916

[28] Nakajima, H., Yoshioka, K.: Instanton counting on blowup. II. K-theoretic partition function. arXiv:math/0505553 
[29] Shchechkin, A.: Blowup relations on $\mathbb{C}^{2} / \mathbb{Z}_{2}$ from Nakajima-Yoshioka blowup relations. arXiv:2006.08582

[30] Tsuda, T.: Tau functions of q-Painlevé III and IV equations. Lett. Math. Phys. 75, 39 (2006)

[31] Alim, M., Cecotti, S., Cordova, C., Espahbodi, S., Rastogi, A., Vafa, C.: $\mathcal{N}=2$ quantum field theories and their BPS quivers. Adv. Theor. Math. Phys. 18, 27 (2014). arXiv:1112.3984

[32] Galakhov, D., Longhi, P., Mainiero, T., Moore, G.W., Neitzke, A.: Wild wall crossing and BPS giants. JHEP 11, 046 (2013). arXiv:1305.5454

[33] Cordova, C.: Regge trajectories in $\mathcal{N}=2$ supersymmetric Yang-Mills theory. JHEP 09, 020 (2016). arXiv:1502.02211

[34] Joshi, N., Nakazono, N., Shi, Y.: Lattice equations arising from discrete painlevé systems. I. $\left(a_{2}+a_{1}\right)^{(1)}$ and $\left(a_{1}+a_{1}^{\prime}\right)^{(1)}$ cases. J. Math. Phys. 56, 092705 (2015). https://doi.org/10.1063/1.4931481

[35] Fomin, S., Zelevinsky, A.: Cluster algebras I: foundations. J. Am. Math. Soc. 15, 497 (2002)

[36] Fomin, S., Zelevinsky, A.: Cluster algebras IV: coefficients. arXiv Mathematics e-prints arXiv:math/0602259 (2006)

[37] Fock, V., Marshakov, A.: Loop groups, clusters, dimers and integrable systems. arXiv:1401.1606

[38] Joshi, N.: Discrete Painlevé Equations, CBMS Regional Conference Series in Mathematics. Conference Board of the Mathematical Sciences (2019)

[39] Okamoto, K.: Sur les feuilletages associés aux équation du second ordre à points critiques fixes de p. painlevé espaces des conditions initiales (1979)

[40] Takenawa, T.: Weyl group symmetry of type $d_{5}^{(1)}$ in the $q$-painlevé v equation. Funkcialaj Ekvacioj 46, 173 (2003)

[41] Kajiwara, K., Nakazono, N., Tsuda, T.: Projective reduction of the discrete Painlevé system of type $\left(A_{2}+A_{1}\right)^{(1)}$. arXiv e-prints arXiv:0910.4439 (2009)

[42] Bonelli, G., Lisovyy, O., Maruyoshi, K., Sciarappa, A., Tanzini, A.: On Painlevé/gauge theory correspondence. Lett. Math. Phys. 107, 2359 (2017). arXiv:1612.06235

[43] Grassi, A., Gu, J.: Argyres-Douglas theories, Painlevé II and quantum mechanics. arXiv: 1803.02320

[44] Huang, M.-X., Sun, K., Wang, X.: Blowup equations for refined topological strings. JHEP 10, 196 (2018). arXiv:1711.09884

[45] Kim, J., Kim, S.-S., Lee, K.-H., Lee, K., Song, J.: Instantons from Blow-up. JHEP 11, 092 (2019). arXiv:1908.11276

[46] Bonnet, G., David, F., Eynard, B.: Breakdown of universality in multicut matrix models. J. Phys. A 33, 6739 (2000). arXiv:cond-mat/0003324

[47] Katz, S.H., Klemm, A., Vafa, C.: Geometric engineering of quantum field theories. Nucl. Phys. B 497, 173 (1997). arXiv:hep-th/9609239

[48] Hollowood, T.J., Iqbal, A., Vafa, C.: Matrix models, geometric engineering and elliptic genera. JHEP 03, 069 (2008). arXiv:hep-th/0310272

[49] Bonelli, G., Maruyoshi, K., Tanzini, A.: Wild Quiver Gauge theories. JHEP 02, 031 (2012). arXiv:1112.1691 
[50] Hatsuda, Y., Marino, M.: Exact quantization conditions for the relativistic Toda lattice. JHEP 05, 133 (2016). arXiv:1511.02860

[51] Bonelli, G., Grassi, A., Tanzini, A.: Seiberg Witten theory as a Fermi gas. Lett. Math. Phys. 107, 1 (2017). arXiv:1603.01174

[52] Sciarappa, A.: Exact relativistic Toda chain eigenfunctions from Separation of Variables and gauge theory. JHEP 10, 116 (2017). arXiv:1706.05142

[53] Iwaki, K., Nakanishi, T.: Exact WKB analysis and cluster algebras. J. Phys. A Math. Theor. 47, 474009 (2014)

[54] Nekrasov, N.A., Shatashvili, S.L.: Quantization of integrable systems and four dimensional Gauge theories. In: Proceedings, 16th International Congress on Mathematical Physics (ICMP09): Prague, Czech Republic, August 3-8, 2009, pp. 265-289 (2009). arXiv:0908.4052

[55] Nakajima, H., Yoshioka, K.: Lectures on instanton counting. In: CRM Workshop on Algebraic Structures and Moduli Spaces Montreal, Canada, July 14-20, 2003 (2003). arXiv:math/0311058

[56] Grassi, A., Gu, J., Mariño, M.: Non-perturbative approaches to the quantum Seiberg-Witten curve. arXiv:1908.07065

[57] Gavrylenko, P., Marshakov, A., Stoyan, A.: Irregular conformal blocks, Painlevé III and the blow-up equations. arXiv:2006.15652

[58] Nekrasov, N.: Blowups in BPS/CFT correspondence, and Painlevé VI. arXiv:2007.03646

[59] Jeong, S., Nekrasov, N.: Riemann-Hilbert correspondence and blown up surface defects. arXiv:2007.03660

[60] Cecotti, S., Del Zotto, M.: Galois covers of $\mathcal{N}=2$ BPS spectra and quantum monodromy. Adv. Theor. Math. Phys. 20, 1227 (2016). arXiv:1503.07485

[61] Coman, I., Pomoni, E., Teschner, J.: From quantum curves to topological string partition functions. arXiv:1811.01978

[62] Coman, I., Longhi, P., Teschner, J.: From quantum curves to topological string partition functions II. arXiv:2004.04585

[63] Cordova, C., Shao, S.-H.: Schur indices, BPS particles, and Argyres-Douglas theories. JHEP 01, 040 (2016). arXiv:1506.00265

[64] Maruyoshi, K., Song, J.: Enhancement of supersymmetry via renormalization group flow and the superconformal index. Phys. Rev. Lett. 118, 151602 (2017). arXiv:1606.05632

[65] Maruyoshi, K., Song, J.: $\mathcal{N}=1$ deformations and RG flows of $\mathcal{N}=2$ SCFTs. JHEP 02, 075 (2017). arXiv:1607.04281

[66] Gaiotto, D., Moore, G.W., Neitzke, A.: Wall-crossing, Hitchin Systems, and the WKB Approximation. arXiv:0907.3987

[67] Fioravanti, D., Gregori, D.: Integrability and cycles of deformed n=2 gauge theory. Phys. Lett. B 804, 135376 (2020)

[68] Elliott, C., Pestun, V.: Multiplicative Hitchin Systems and Supersymmetric Gauge Theory. arXiv:1812.05516

[69] Bonelli, G., Tanzini, A.: Hitchin systems, $\mathrm{N}=2$ gauge theories and W-gravity. Phys. Lett. B 691, 111 (2010). arXiv:0909.4031 
[70] Bonelli, G., Maruyoshi, K., Tanzini, A.: Quantum Hitchin systems via $\beta$-deformed matrix models. Commun. Math. Phys. 358, 1041 (2018). arXiv:1104.4016

[71] Bonelli, G., Del Monte, F., Gavrylenko, P., Tanzini, A.: $\mathcal{N}=2^{*}$ gauge theory, free fermions on the torus and Painlevé VI. Commun. Math. Phys. 377, 1381 (2020). arXiv:1901.10497

[72] Bonelli, G., Del Monte, F., Gavrylenko, P., Tanzini, A.: Circular quiver gauge theories, isomonodromic deformations and $W_{N}$ fermions on the torus. arXiv:1909.07990

[73] Mironov, A., Morozov, A.: q-Painlevé equation from Virasoro constraints. Phys. Lett. B 785, 207 (2018). arXiv:1708.07479

[74] Nedelin, A., Zabzine, M.: q-Virasoro constraints in matrix models. JHEP 03, 098 (2017). arXiv:1511.03471

[75] Lodin, R., Popolitov, A., Shakirov, S., Zabzine, M.: Solving q-Virasoro constraints. Lett. Math. Phys. 110, 179 (2020). arXiv:1810.00761

[76] Bonelli, G., Grassi, A., Tanzini, A.: New results in $\mathcal{N}=2$ theories from nonperturbative string. Annales Henri Poincare 19, 743 (2018). arXiv:1704.01517

[77] Nekrasov, N.A.: Seiberg-Witten prepotential from instanton counting. In: International Congress of Mathematicians (ICM 2002) Beijing, China, August 20-28, 2002 (2003). arXiv:hep-th/0306211

[78] Bruzzo, U., Fucito, F., Morales, J.F., Tanzini, A.: Multiinstanton calculus and equivariant cohomology. JHEP 05, 054 (2003). arXiv:hep-th/0211108

Giulio Bonelli and Alessandro Tanzini

International School of Advanced Studies (SISSA)

via Bonomea 265

34136 Trieste

Italy

e-mail: bonelli@sissa.it

and

Institute for Geometry and Physics (IGAP)

via Beirut 2/1

34151 Trieste

Italy

and

INFN, Sezione di Trieste

Trieste

Italy

Fabrizio Del Monte

Centre de Recherches Mathématiques (CRM)

Université de Montréal

C. P. 6128succ. Centre Ville

Montréal QCH3C 3J7

Canada

e-mail: delmonte@crm.umontreal.ca 
Communicated by Ruben Minasian.

Received: November 24, 2020.

Accepted: February 15, 2021. 